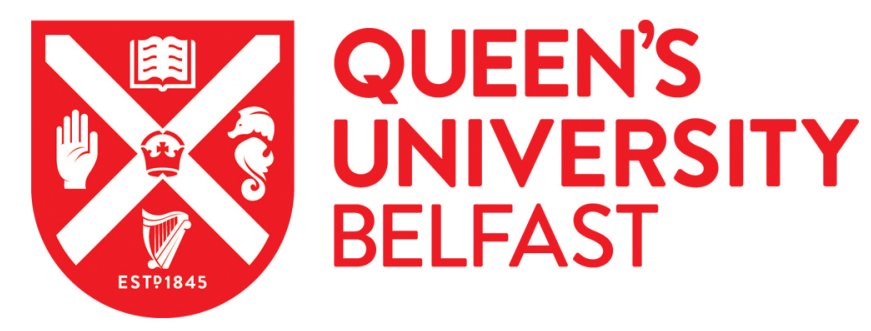

\title{
Microneedle characterisation: the need for universal acceptance criteria and GMP specifications when moving towards commercialisation
}

Lutton, R. E. M., Moore, J., Larrañeta, E., Ligett, S., Woolfson, A. D., \& Donnelly, R. F. (2015). Microneedle characterisation: the need for universal acceptance criteria and GMP specifications when moving towards commercialisation. Drug Delivery and Translational Research, 5(4), 313-331. https://doi.org/10.1007/s13346015-0237-z

Published in:

Drug Delivery and Translational Research

Document Version:

Peer reviewed version

Queen's University Belfast - Research Portal:

Link to publication record in Queen's University Belfast Research Portal

Publisher rights

(c) Controlled Release Society 2015

The final publication is available at Springer via http://link.springer.com/article/10.1007\%2Fs13346-015-0237-z

\section{General rights}

Copyright for the publications made accessible via the Queen's University Belfast Research Portal is retained by the author(s) and / or other copyright owners and it is a condition of accessing these publications that users recognise and abide by the legal requirements associated with these rights.

\section{Take down policy}

The Research Portal is Queen's institutional repository that provides access to Queen's research output. Every effort has been made to ensure that content in the Research Portal does not infringe any person's rights, or applicable UK laws. If you discover content in the

Research Portal that you believe breaches copyright or violates any law, please contact openaccess@qub.ac.uk. 


\section{Microneedle characterisation: The need for universal acceptance criteria and GMP specifications when moving towards commercialisation.}

Rebecca E.M. Lutton, Jessica Moore, Eneko Larrañeta, Stephen Ligett, A. David Woolfson, Ryan F. Donnelly*.

Queens University, Belfast School of Pharmacy, 97 Lisburn Road, Belfast BT9 7BL, United Kingdom

*Corresponding author at: Chair in Pharmaceutical Technology, School of Pharmacy, Queens University Belfast, Medical Biology Centre, 97 Lisburn Road, Belfast BT9 7BL, United Kingdom. Tel.: +44 2890972 251; fax: +44 2890247 794. E-mail address: r.donnelly@qub.ac.uk (R.F. Donnelly).

Keywords: Microneedles; Characterisation; Quality Control; CGMP; Specifications; Commercialisation.

\section{Contents}

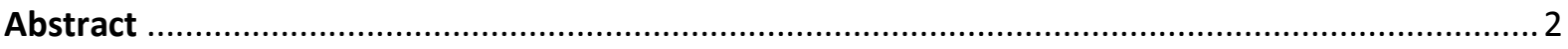

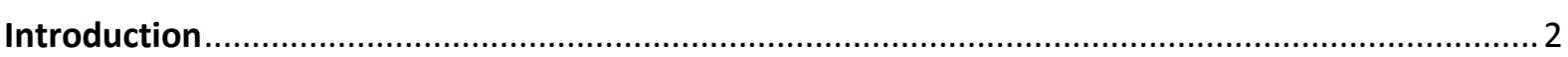

1. Good Manufacturing Practice Standard Specifications ............................................................ 4

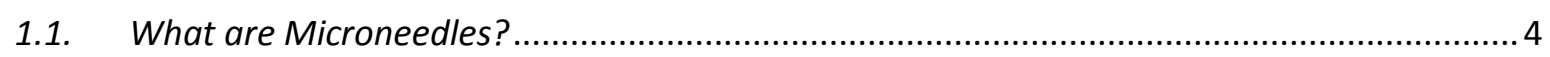

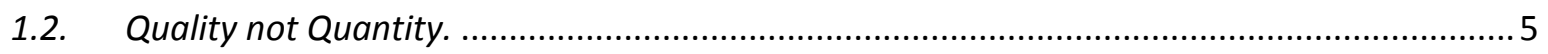

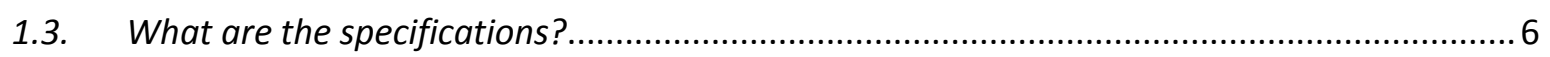

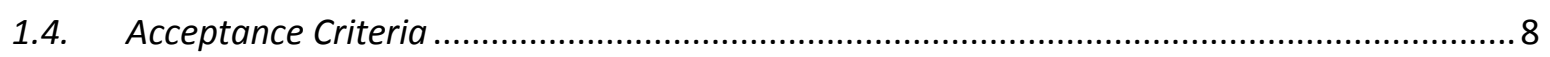

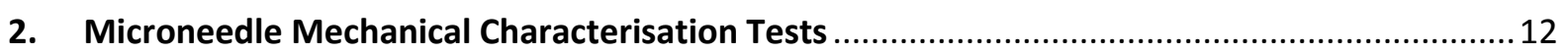

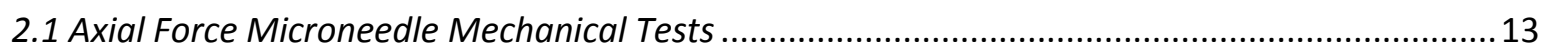

2.2. Transverse Force and Shear Strength Microneedle Mechanical Tests.......................................13

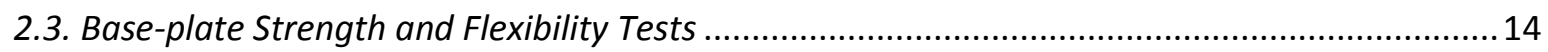

2.4. Significance of Microneedle Mechanical Test Results............................................................. 14

3. Techniques Used to Analyse Microneedle Insertion.............................................................. 16

3.1. Staining of Microneedle-Treated Skin .................................................................................... 16

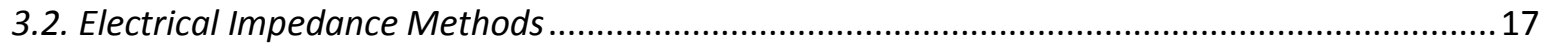

3.3. Transepidermal Water Loss (TEWL) Monitoring .................................................................. 18

3.4. Histological Tissue Staining and Sectioning …................................................................... 19

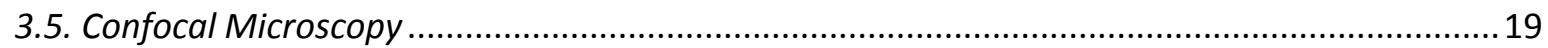

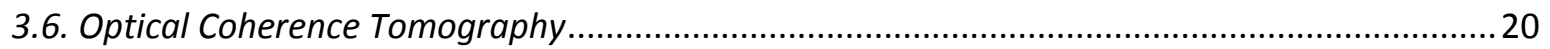

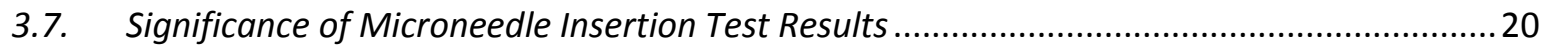

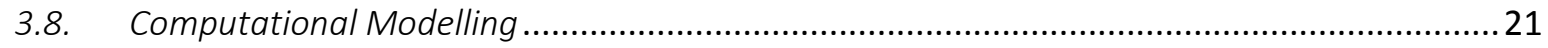

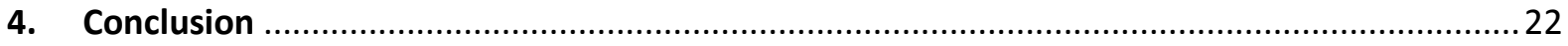




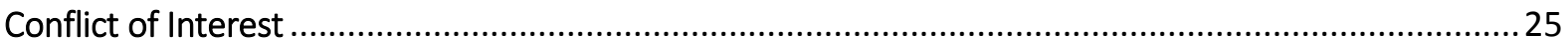

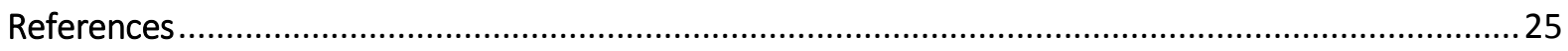

\begin{abstract}
With interest in microneedles as a novel drug transdermal delivery system increasing rapidly since the late 1990's [1], a diverse range of microneedle systems have been fabricated with varying designs and dimensions. However, there are still very few commercially available microneedle products. One major issue regarding microneedle manufacture on an industrial scale is the lack of specific quality standards for this novel dosage form in the context of Good Manufacturing Practice (GMP). A range of mechanical characterisation tests and microneedle insertion analysis techniques are used by researchers working on microneedle systems to assess the safety and performance profiles of their various designs. The lack of standardised tests and equipment used to demonstrate microneedle mechanical properties and insertion capability makes it difficult to directly compare the in use performance of candidate systems. This review highlights the mechanical tests and insertion analytical techniques used by various groups to characterise microneedles. This in turn exposes the urgent need for consistency across the range of microneedle systems in order to promote innovation and the successful commercialisation of microneedle products.
\end{abstract}

\title{
Introduction
}

Transdermal drug delivery (TDD) is the systemic delivery of drugs through the skin, typically via the use of adhesive skin patches. A major advantage of TDD systems is the ability to release drug over an extended time period. Thus, systemic drug concentration can be maintained within the desired therapeutic window for a longer time. This also extends the activity of drugs that have short half-lives due to their rapid metabolism. The transdermal route avoids problems in drug absorption in the gastrointestinal tract and hepatic first-pass effects. Furthermore, the convenience and non-invasive nature of TDD offers improved patient acceptability and compliance. The TDD market, worth $\$ 12.7$ billion dollars in 2005, is expected to reach $\$ 32$ billion in 2015 and as such, has been an important area of pharmaceutical research and development for the last few decades $[1,2,3,4]$.

Due to the need for passive diffusion through the stratum corneum (SC), the skin's outer layer that provides a barrier to the external environment, there are selection restrictions on the choice of drugs amenable to TDD. Drugs currently administered by TDD share three features that enable administration through a convenient area of skin: molecular mass $<500 \mathrm{Da}$; high lipophilicity and low 
required daily dose ( $<2 \mathrm{mg}$ ) [1]. These physicochemical imitations to TDD, imposed by the nature of the skin barrier, have led to investigations for alternative TDD methods.

One area of TDD research that shows considerable promise in overcoming the physicochemical barriers inherent in the route is that of microneedles (MNs). MNs are arrays consisting of multiple MN projections (ranging from 50-900 $\mu \mathrm{m}$ in height) [5]. Their micron scale allows them to successfully bypass the SC, without stimulating nociceptors (pain receptors) in the underlying epidermal/dermal tissue layers $[5,6,7,8]$. Over the past decade, an extensive range of potential MN applications have been proposed. Their prospective applications have varied from the use of MNs to enhance transdermal delivery of biomolecules (therapeutic peptides, proteins and vaccine antigens), to their incorporation into sophisticated automated closed-loop systems for blood glucose testing and delivery of insulin [9].

Different groups have investigated different types of microneedles, from in-plane [10] and out-ofplane [11], to hollow [12], solid [13], macroporous [14], dissolving and swelling [15]. They have been produced from a variety of materials such as glass [16], sugar [17], metal [18], metal coated [19], silicon [14], solid polymer [20], aqueous hydrogel [21] and dissolving polymers [15]. MN have been fabricated using a diversity of techniques, mostly from microelectromechanical systems (MEMS) technology. Fabrication techniques range from ion sputtering deposition [22], photolithography [23], wet and dry etching [14], photopolymerisation [24], laser ablation and micromoulding [25,26], layerby-layer deposition [27], droplet-born air blowing [28], and milling [29]. With such a collection of MN types and fabrication techniques, and with most having experienced some degree of success, it is surprising there are still very few MN products on the market. In fact, some may not class the current commercial $\mathrm{MN}$ products as the most optimal microneedle products nor do they reflect the original, broader scope for MNs in their truest sense: simple products which may be applied in patch form, easily, painlessly, economically, and requiring no specialist training or specialised waste disposal units. Figure 1 displays the current $M N$ systems either on the market $(B, G$ and $H$ ) or awaiting approval for market release.: the Microstructured Transdermal System (3M), Microinfusor (BD), Macroflux ${ }^{\circledR}$ (Alza), Microneedle Therapy system (MTS Roller ${ }^{\mathrm{TM}}$; Clinical Resolution Lab) and, Micro-trans ${ }^{\mathrm{TM}}$ and h-patch ${ }^{\mathrm{TM}}$ (Valeritas). Nevertheless, despite the potential conflict with the original MN ideal, these products do achieve the main aim at the heart of TDD research: painlessly by-passing the SC with potential for drug delivery, eliminating the need for the traditional hypodermic needle.

It is evident that there is a clear market for successful mass production of MNs. However, due to the difficulty in scale-up of fabrication, this has sadly not been fully exploited as yet. There are no MNs on the market that embrace the broad scope of ideal objectives, nor even reflect the current research 
activity. Some products, marketed as MNs are not MNs as such but rather are best described as very short hypodermic needles. Consequently, there are no accepted regulatory standards in place for true MN products. This creates further complex problems regarding mass manufacture, which requires accepted standards by which to assess product quality. This is a significant obstacle in the path of MN commercialisation.

The intention of this review is not to explore the variety of materials suitable for microneedles, nor the diversity of production methods used in their fabrication. Instead, the purpose is to convey the need for standardised quality control tests to characterise and investigate the structural integrity of fully formed microneedles at the final stage of mass production. The need for a universal acceptance criteria and GMP specifications is deemed necessary, and a high priority, due to the vast array of research currently being conducted in this area and the emergence of products with similar applications, already on the marketplace.

\section{Good Manufacturing Practice Standard Specifications}

Laboratory-based processes are often difficult to scale-up initially, with problems of cost-efficiency of mass manufacture and turnaround time. Rapid turnaround may be required, for example, where there is a an urgent need for products in a pandemic situation like the 2009 H1N1 swine flu pandemic, the recent outbreaks of Avian influenza $A(H 5 N 1)$ and more recently $A(H 7 N 9)$ and the 2014 Ebola crisis.

Once a method of cost-efficient mass manufacture, with large product volume turnover, has been developed, it will need to be capable of being adopted into Good Manufacturing Practice (GMP) protocols and guidelines. This in itself presents significant issues and complications.

\subsection{What are Microneedles?}

Section 501(a)(2)(B) of the Federal Food, Drug, and Cosmetic Act (FD\&C Act) requires drugs, which include Investigational New Drug (IND) products, to comply with current good manufacturing practice (cGMP) as follows [30]:

A drug...shall be deemed adulterated...if...the methods used in, or the facilities or controls used for, its manufacture, processing, packing, or holding do not conform to or are not operated or administered in conformity with current good manufacturing practice to assure that such drug meets the requirements of this Act as to safety and has the identity and strength, and meets the quality and purity characteristics, which it purports or is represented to possess. 
This poses a key question: what exactly are microneedles? How do we define them? This excerpt requires that any new drug product has defined identity, strength, quality and purity. It may be thought that this only applies to drug substances and that as a result does not apply to parenteral drug products such as MNs, however, this is mistaken. This leads to a further question: are microneedles drug products, parenteral products or devices? Does it even matter? The answer may be both yes and no.

It may be argued that microneedles branch both categories. Dissolving microneedles [31], whereby the MNs are made from drug-loaded, dissolving polymeric matrices, could be classed with other dissolving or disintegrating drug products, such as tablets. The likes of hydrogel-forming [21] or metal [19] microneedles, where the arrays are removed from the skin intact after drug delivery, may be classed alongside other parenteral products or medical devices. However, it could be that they are all classed under the umbrella of 'drug product'. The difference and distinction becomes important when the manufacturer wishes to commercially produce the product; they will need to know which quality standards the product must conform to. The problem, of course, is that there are no standards set for MNs at present due to there being no true MN products on the market.

\subsection{Quality not Quantity.}

The holder of a Manufacturing Authorisation must manufacture medicinal products so as to ensure that they are fit for their intended use, comply with the requirements of the Marketing Authorisation or Clinical Trial Authorisation, as appropriate, and do not place patients at risk due to inadequate safety, quality or efficacy. Article 6 of Directives 2003/94/EC and 91/412/EEC require manufacturers to establish and implement an effective pharmaceutical quality assurance system. In order to achieve this quality objective reliably there must be a comprehensively designed and correctly implemented Pharmaceutical Quality System incorporating Good Manufacturing Practice and Quality Risk Management [32]. Finished product assessment should embrace all relevant factors, including production conditions, results of in-process testing, a review of manufacturing (including packaging) documentation, compliance with the Finished Product Specification and visual examination of the final finished pack.

For industrial manufacture, production conditions can be easily documented and consistently repeatable due to automation; this is particularly the case for large scale manufacture as would be expected of MNs for vaccine use. In-process quality control tests testing are those that may be performed during the manufacture of either the drug substance or drug product, rather than as part of the tests conducted prior to release [33]. Current in-line product inspection techniques include contaminant detection by $\mathrm{x}$-ray, motion check weighing systems and white light scanner systems 
allowing comprehensive 3D optical measurements. Again, the issue arises in knowing what, specifically, to be inspecting. At this stage of the process it will probably depend primarily on the MN type. Equally, it must be remembered that, in some instances, the final microneedle product is not the same as that at release, an example being MNs requiring a backing layer(s) to be applied to form a MN-based patch system.

An important part of the required documentation deals with Quality Control, of which specifications, particularly the finished product specifications, play a substantial role [34]. It is understood that there will be large demand for high throughput in mass manufacture, particularly for such a functional product. However, it is precisely for this reason that quality control is paramount. Quality Control is concerned with sampling, specifications and testing as well as the organisation, documentation and release procedures designed to ensure that the necessary and relevant tests are carried out, and that materials are not released for use, nor products released for sale or supply, until their quality has been judged satisfactory. Quality Control is not confined to laboratory operations, but must be involved in all decisions that may concern the quality of the product. The independence of Quality Control from Production is considered fundamental to the satisfactory operation of a Quality Control System $[32,34]$. Again, the problem for MN manufacture arises from the lack of known and agreed product specifications. MN researchers need to agree upon putative universal acceptance criteria for MN specifications. What are suitable and credible tests that are simple and quick to perform and which yield sufficient data to determine that the quality of the product is of the required standard?

\subsection{What are the specifications?}

The International Conference on Harmonisation (ICH) Harmonised Tripartite Guideline Specifications: Test Procedures and Acceptance Criteria for New Drug Substances and New Drug Products: Chemical Substances Q6A, is a guideline intended to assist in the establishment of a single set of global specifications for new drug substances and new drug products. It provides guidance on the setting and justification of acceptance criteria and the selection of test procedures for new drug substances of synthetic chemical origin, and new drug products produced from them, which have not been registered previously in the United States, the European Union, or Japan. The quality of drug substances and drug products is determined by their design, development, in-process controls, GMP controls, and process validation, and by specifications applied to them throughout development and manufacture. This particular guideline addresses specifications, i.e., those tests, procedures, and acceptance criteria that play a major role in assuring the quality of the new drug substance and/or new drug product at release and during shelf life. Specifications are an important component of an overall Quality Assurance system (of which Quality control forms a part), but are not its only 
component. All of the above considerations are necessary to ensure consistent production of drug substances and drug products of high quality [33].

The ICH Q6A state:

A specification is defined as a list of tests, references to analytical procedures, and appropriate acceptance criteria, which are numerical limits, ranges, or other criteria for the tests described. It establishes the set of criteria to which a drug substance or drug product should conform to be considered acceptable for its intended use. "Conformance to specifications" means that the drug substance and/or drug product, when tested according to the listed analytical procedures, will meet the listed acceptance criteria. Specifications are critical quality standards that are proposed and justified by the manufacturer and approved by regulatory authorities as conditions of approval. Specifications are one part of a total control strategy for the drug substance and drug product designed to ensure product quality and consistency. Other parts of this strategy include thorough product characterization during development, upon which specifications are based, and adherence to Good Manufacturing Practices; e.g., suitable facilities, a validated manufacturing process, validated test procedure, raw material testing, in-process testing, stability testing, etc. Specifications are chosen to confirm the quality of the drug substance and drug product rather than to establish full characterization, and should focus on those characteristics found to be useful in ensuring the safety and efficacy of the drug substance and drug product.

The guidelines [33] also define a new drug product as a pharmaceutical product type, for example, tablet, capsule, solution, cream, etc., which has not previously been registered in a region or Member State, and which contains a drug ingredient generally, but not necessarily, in association with excipients. It may be assumed on this basis that the MN types previously stated, which may fall into the parenteral category, would be exempt from requirement. However, this is not the case, as the following section makes clear.

Dosage forms addressed in this guideline include solid oral dosage forms, liquid oral dosage forms, and parenteral (small and large volume). The extended application of the concepts in this guideline to other dosage forms, e.g., to inhalation dosage forms (powders, solutions, etc.), to topical formulations (creams, ointments, gels), and to transdermal systems, is encouraged. 
It is clear, therefore, that MNs fall within the scope of the ICH Q6A and thus, that a set of quality specifications for MNs, ideally applicable to all types, is required. This would certainly aid in comparative research and quicken the pace at which $\mathrm{MN}$ research is translated from laboratory to patient. In order to achieve this, though, it is important to know the desired criteria on which a product specification should be based.

When a specification is first proposed, justification should be presented for each procedure and each acceptance criterion should be included. The justification should refer to relevant development data, pharmacopoeial standards (where available), test data for drug substances and drug products used in toxicology and clinical studies, and results from accelerated and long term stability studies, as appropriate. Additionally, a reasonable range of expected analytical and manufacturing variability should be considered. The applicant should justify alternative approaches. Such justification should be based on data derived from the new drug product manufacturing process. This justification may consider theoretical tolerances for a given procedure or acceptance criterion. If multiple manufacturing sites are planned, it may be valuable to consider data from these sites in establishing the initial tests and acceptance criteria [33].

\subsection{Acceptance Criteria}

It is recognized that only a limited amount of data may be available at the time of filing, which can influence the process of setting acceptance criteria. The basis for the acceptance criteria at the time of filing should necessarily focus on safety and efficacy [33].

It is this last point which is at the heart of the question of MN specification criteria: safety and efficacy. What are the basic requirements of MNs? They need to pierce the skin, penetrate, remain intact or dissolve $\mathrm{n}$ the skin, whilst delivering the drug cargo, then be removed, still intact, or if dissolving, have done so within the required timeframe; all this whilst remaining harmless to the patient.

The required specific tests and criteria for both new drug products and parenteral drug products, which could be feasibly applied to MNs, could be merged and adopted from the ICH Harmonised Tripartite Guidelines. If this were to be done then the following would be obligatory and could form the basis of a structure for set of universal acceptance criteria for MN specifications:

a) Dissolution: A test to measure release of drug substance from the drug product. If changes in formulation or process variables significantly affect dissolution and such changes are not controlled by another aspect of the specification, it may also be appropriate to adopt dissolution test conditions that can distinguish these changes. This is potentially important for processes whereby the MNs change during the process, such as in photopolymerisation or crosslinking. 
b) Disintegration: For rapidly dissolving (dissolution $>80 \%$ in 15 minutes at $\mathrm{pH} 1.2,4.0$ and 6.8) products disintegration may be substituted for dissolution. Disintegration testing is most appropriate when a relationship to dissolution has been established or when disintegration is shown to be more discriminating than dissolution. MNs designed to dissolve in the skin, thereby releasing their drug loading, may fall into this category of test.

c) Hardness/friability: It is normally appropriate to perform hardness and/or friability testing as an in-process control. If the characteristics of hardness and friability have a critical impact on drug product quality (e.g., chewable tablets), acceptance criteria should be included in the specification. This last point may be pertinent to swelling MNs: can they still be removed intact after swelling? A simple test such as infra-red spectroscopy (which can determine crosslinked bonds in polymeric MN) may be all that it is required [35].

d) Uniformity of dosage units: This harmonised pharmacopoeial test includes the mass of the dosage form and the content of the active substance in the dosage form, as appropriate. Again it needs to be highlighted that, in some cases, MN production may result in an intermediate stage of a final product, i.e., there may be no drug content at this stage and thus the test would apply to the final assembled product.

e) Water content: A test for water content should be included when appropriate. The acceptance criteria may be justified with data on the effects of hydration or water absorption on the drug product. In some cases, a Loss on Drying procedure may be considered adequate; however, a detection procedure which is specific for water (e.g., Karl Fischer titration) is preferred. This will be essential for dehydrated/hygroscopic MN forms.

f) Microbial limits: Microbial limit testing is seen as an attribute of Good Manufacturing Practice. In general, it is advisable to test the drug product unless its components are tested before manufacture and the manufacturing process is known, through validation studies, not to carry a significant risk of microbial contamination or proliferation. As MN differ from conventional TDS in that they penetrate the skin rather than remain on its surface, this point is particularly relevant, whether in terms of a requirement for a sterile or a low bioburden product. Conventional parenteral products typically form into the former category but some polymeric $\mathrm{MN}$ do not appear to support microbial growth.

g) Sterility: All parenteral products should have a test procedure and acceptance criterion for evaluation of sterility. Where data generated during development and validation justify parametric release, this approach may be proposed for terminally sterilized drug products. 
h) Particulate matter: Parenteral products should have appropriate acceptance criteria for particulate matter. This will normally include acceptance criteria for visible particulates and / or clarity of solution, as well as for sub-visible particulates as appropriate.

i) Antimicrobial preservative content: For parenteral products needing an antimicrobial preservative, acceptance criteria for preservative content should be established. Testing for antimicrobial preservative content should normally be performed at release. Under certain circumstances, in-process testing may suffice in lieu of release testing where permitted. When antimicrobial preservative content testing is performed as an in-process test, the acceptance criteria should remain part of the specification. Antimicrobial preservative effectiveness should be demonstrated during development, during scale up, and throughout the shelf-life. It seems doubtful that use of an antimicrobial preservative will be appropriate for $\mathrm{MN}$ products.

j) Extractables: Control of extractables from container/closure systems is considered significantly more important for parenteral products than for oral liquids. However, where development and stability data show evidence that extractables are consistently below the levels that are demonstrated to be acceptable and safe, elimination of this test can normally be accepted. This should be reinvestigated if the container/closure system or formulation changes.

k) Functionality testing of delivery systems: Parenteral formulations packaged in prefilled syringes, autoinjector cartridges, or the equivalent should have test procedures and acceptance criteria related to the functionality of the delivery system. For $\mathrm{MN}$, a key functionality test may be based on assessing their ability to penetrate the skin throughout the shelf life of the product.

I) Osmolarity: When the tonicity of a product is declared in its labelling, appropriate control of its osmolarity should be performed. This will not be relevant to MN systems.

One final aspect, mentioned in both these guidelines and also in the EU Guidelines for Good Manufacturing Practice for Medicinal Products for Human and Veterinary Use Quality Control document [34], is the need for an on-going stability programme. After marketing, the stability of the medicinal product should be monitored according to a continuous appropriate programme that will permit the detection of any stability issue (e.g., changes in levels of impurities or dissolution profile) associated with the formulation in the marketed package. The purpose of the on-going stability programme is to monitor the product over its shelf life and to determine that the product remains, and can be expected to remain, within specifications under the labelled storage conditions. This mainly applies to the medicinal product in the package in which it is sold, but consideration should also be given to the inclusion in the programme of bulk product. For example, when the bulk product is stored for a long period before being packaged and/or shipped from a manufacturing site to a packaging site, the impact on the stability of the packaged product should be evaluated and studied 
under ambient conditions. In addition, consideration should be given to intermediates that are stored and used over prolonged periods. Stability studies on reconstituted product are performed during product development and need not be monitored on an on-going basis. However, when relevant, the stability of a reconstituted product can also be monitored. The protocol for an on-going stability programme should extend to the end of the shelf life period and should include, but not be limited to, the following parameters: relevant physical, chemical, microbiological and biological test methods. The required information includes the i) Acceptance criteria; ii) Reference to test methods; iii) Description of the container closure system(s); iv) Testing intervals (time points). Unless otherwise justified, at least one batch per year of product manufactured in every strength and every primary packaging type, if relevant, should be included in the stability programme. For MN systems, particularly those derived from aqueous polymer solutions or dispersions, the ability of the MN to retain their inherent shape and strength, and to avoid absorbing atmospheric moisture during storage, is an additional aspect to the stability of any drug component present in the product.

It is recognised that only a limited amount of data may be available at the time of filing, which can influence the process of setting acceptance criteria [33]. As previously mentioned, the basis for the acceptance criteria at the time of filing should necessarily focus on safety and efficacy. Equally, evolving new analytical technologies, and modifications to existing technology, are continually being developed. With the further development and future commercialisation of MNs, such technologies should be used when they are considered to offer additional assurance of quality, or are otherwise justified. It must be noted, however, that any amendments to existing manufacturing processes or development of new techniques need to be re-evaluated in accordance with qualification and validation guidelines [36]. Additionally, migration of an already qualified and validated process to a clean room must similarly be evaluated against the required guidelines [37]. This is data that can help advance $\mathrm{MN}$ research at a quicker pace than current progress and increase the prospect of commercialisation in the near future.

The following statement in the legislation guidelines is particularly interesting with respect to novel product development.

GMP applies to the lifecycle stages from the manufacture of investigational medicinal products, technology transfer, commercial manufacturing through to product discontinuation. However the Pharmaceutical Quality System can extend to the pharmaceutical development lifecycle stage, which while optional, should facilitate innovation and continual improvement and strengthen the link between pharmaceutical development and manufacturing activities [32] 
In order to comply with this statement, there needs to be universal agreement on the desired criteria for MN testing. Laboratory tests used in manufacturing (e.g., testing of materials, in-process material, packaging, drug product) should be scientifically sound (e.g., specific, sensitive, and accurate), suitable and reliable for the specified purpose. Testing should evaluate quality attributes appropriate to the product [30].

Part of the definition of specifications is to include 'thorough product characterization during development, upon which the specifications are based' and one of the desired specifications for parenteral new drug products is 'functionality testing of delivery systems' [33]. Researchers are aware of the need for characterisation and analysis of novel products under development research groups create tests and assume these as internal specifications and standards. This is appropriate for general laboratory protocols and comparison within a group or cluster of candidate systems; however, this produces a problem when external groups wish to compare their results. Thus, a review of characterisation and insertion techniques used by various research groups is described in the following sections.

\section{Microneedle Mechanical Characterisation Tests}

Mechanical characterisation of MNs is a crucial step in the development of successful MNs. MNs are subjected to a diverse range of stresses, including those experienced on insertion due to nonuniformity of the skin surface, unavoidable movements during insertion and the stresses exerted upon removal. MNs therefore need to possess a standard inherent strength to avoid unacceptable MN array failure due to these stresses [38,39]. These stresses can cause failure by a number of modes including MN bending, buckling and base-plate fracturing [40]. There is no single test that can simulate insertion of $\mathrm{MN}$ in vivo and fully characterise mechanical performance; therefore, characterisation consists of a range of tests.

One of the first, and more basic, MN mechanical tests (Figure 2) to be demonstrated was by Zahn et al. [40]. The test involved the base of a single, hollow, polysilicon MN attached to a glass slide, held in place by a clamp, while a gradually increasing vertical force being exerted at the MN tip by a force gauge (0-20 g range) until it fractures. Further investigations were made of whether coating the MNs with either nickel, platinum or titanium improved needle strength, with the latter considered the most biocompatible. The methodology was flawed in that the MNs were coated with 10,10 and $5 \mu \mathrm{m}$ of each respective metal; therefore, the enhanced strength acquired from the metal coatings cannot be directly compared. Overall, predictions of $\mathrm{MN}$ strength were overestimated when compared with experimental results. This was possibly due to polysilicon being a ceramic material. Thus, any flaws in the MN surface would cause crack initiation and propagation, which were not considered. 


\subsection{Axial Force Microneedle Mechanical Tests}

Axial force mechanical tests are commonly employed by $\mathrm{MN}$ groups to assess the mechanical strength of MNs. Axial compression tests involve subjecting the MNs to a force perpendicular to the base-plate [26]. They typically involve the use of a mechanical test station which records both displacement and force while the MNs are pushed against a hard metallic surface at a defined rate $[41,42,43]$, Figure $3 \mathrm{~A}$. Analysis of the force-displacement curves generated show a sudden decrease in force upon MN fracture; the maximum force exerted immediately prior to this drop is usually taken to be the MN failure force $[42,44]$. MN groups performing these tests typically image the MNs before and after to validate fracture and possibly determine the mode of failure for their particular MNs; Gittard et al. [45] found the main mode of failure of acrylate-based polymeric MNs was via compression, followed by MN bending.

Fracture force data from Davis et al. [44], similarly to Zahn et al. [40] and Khann et al. [39], should be viewed with caution, as the experiments involved mechanically characterising single MNs. As with the effect of $\mathrm{MN}$ density on insertion, as discussed, the failure force of a $\mathrm{MN}$ array cannot be assumed to be the same as that of a single MN. It is also important to mention that the force exerted on the MNs during the compression studies does not accurately simulate the forces the MNs experience on insertion into the skin. In compression studies, where MNs are pressed against a hard metallic surface, the whole force exerted is concentrated on the MN tip contact surface. However, the forces used to insert the MNs into the skin are distributed over a greater $M N$ area, especially following initial penetration, as the flexible skin wraps around the MN projections [45].

\subsection{Transverse Force and Shear Strength Microneedle Mechanical Tests}

Irregularity of the skin surface often leads to incomplete insertion of the $\mathrm{MN}$ array and can cause transverse bending of the MNs. Transverse fracture force tests are therefore necessary to provide a more complete profile of the behaviour of MNs during application [42,43]. A mechanical test station is commonly used, where a transverse force (force applied parallel to the $\mathrm{MN}$ base-plate and normal to the microneedle $\mathrm{y}$-axis) is applied at a defined point of the $\mathrm{MN}$ via a metal probe until the $\mathrm{MN}$ fractures. Analysis of the force-displacement curves will identify the fracture force. A sudden drop in force indicates $M N$ failure $[26,43]$. Transverse fracture force testing of a row of MNs in an array, rather than a single $\mathrm{MN}$, involves dividing the force required to fracture all the MNs within the row by the number of MNs, to calculate the transverse fracture force per individual MN [26]. A notable limitation of this test is the fact that it requires the researcher to manually align the metal probe with a defined length on the MN [26]. This is difficult considering the micron scale of $M N$ and so may introduce experimental inaccuracies, although a microscope camera can be used to aid alignment [43]. 
Khanna et al. [39] highlighted the need for shear fracture characterisation due to lateral forces acting on MN arrays. They investigated the effect of geometry on MN shear fracture strength. This study involved comparison of the shear strength of 'I' shaped lumen and circular lumen silicon MNs, Figure 4. As expected, the shear fracture limits of the ' $I$ ' shaped MNs were consistently higher than the circular MNs in the lateral direction (Y-axis), due to the larger second moment of inertia, and were almost similar in the transverse direction (X-axis). The authors commented that this can be of advantage for MNs when greater shear force is expected in a particular direction, however, they do not comment on what situation would produce such an event. Equally, there were no insertion studies. This shape may be difficult to mass produce also to insert effectively into skin.

\subsection{Base-plate Strength and Flexibility Tests}

Assessment of the MN base-plate strength is important as, regardless of the MN projections' resilience, fracturing of the base-plate on patient application is not acceptable. The base-plate needs to possess a degree of flexibility to conform to the non-uniform topography of the skin without fracturing [26]. Donnelly et al. [26] used mechanical tests to investigate the break strength and flexibility of polymeric base-plates. A mechanical test station incorporating a Texture Analyzer was used, where the base-plate was placed between two aluminium blocks and a metal probe moved towards the base-plate at a speed of $2 \mathrm{~mm} / \mathrm{s}$, with a $5 \mathrm{~mm}$ maximum distance to travel. The peak maximum observed in the force-distance curve generated represented the force required to break the base-plate $(2.38 \pm 0.54 \mathrm{~N})$, while the angle of base-plate bending upon breaking was used to evaluate base-plate flexibility $\left(1.28 \pm 0.21^{\circ}\right)$. A schematic illustration of this experiment is presented in Figure 5.

\subsection{Significance of Microneedle Mechanical Test Results}

The significance of the experimentally determined $\mathrm{MN}$ failure forces can only be properly evaluated when compared with the corresponding insertion forces [42]. 'Margin of safety' is a useful index value used by a number of $M N$ groups to express the ratio of $M N$ fracture force: insertion force $[41,42,44]$. Subsequently, in order to measure the insertion forces for MN arrays human or animal a skin sample is required. The majority of reported studies use biological tissue, normally human cadaver skin or animal skin. In these studies, the piercing force was not determined directly; instead what was evaluated was the ability to pierce, or not, the skin of different MN arrays using microscopy, histological analysis or alternative techniques.

Only a few research groups directly measured the insertion forces of $M N$ in the skin. Davis et al. [44] performed a study with human volunteers measuring the insertion forces of metal MN with tip radii varying between 30 and $80 \mu \mathrm{m}$ using a force-displacement measurement device. The obtained values 
for these forces ranged between 0.08 and $3.04 \mathrm{~N}$ per needle, values that permit insertion by hand. Park [46] , following the approach of Davis et al., measured the insertion forces of tapered and solid microneedles. The measured insertion forces were 0.8 and $1.29 \mathrm{~N}$ for MN having tip diameters of 55 and $115 \mu \mathrm{m}$, respectively. Alternatively Loeters et al. [47] developed a method to evaluate microneedle penetration using microneedles with integrated electrodes. In this way penetration could be verified by electrical measurements [42]. The penetration force needed for piercing a porcine skin sample using a $9 \times 9$ microneedle array in vitro was $2.6 \mathrm{~N}$. Moreover, Roxhed et al. [48] measured the insertion forces of ultrasharp microneedles (tip radius of $0.1 \mu \mathrm{m}$ ) in human volunteers using electrical impedance measurements in the skin. The obtained values were lower than $10 \mathrm{mN}$.

Recently Khanna et al. [9] measured the insertion forces of different $4 \times 4$ hollow silicon MN arrays in human cadaver skin using force-displacement measurement. The obtained insertions forces ranged between $4.75 \mathrm{~N}$ and $0.1 \mathrm{~N}$, depending on the sharpness of the tips.

All MN arrays should possess a fracture force greater than the force required for insertion into the patient's skin. Therefore, a MN array with a failure force less than the force needed for insertion would 'fail', as the MNs would fracture upon insertion. MN arrays can be optimised to increase the 'margin of safety' value; Park et al. [42] found a decrease in MN height was associated with a desirable increase in the 'margin of safety' from 1.7 to 3.8. The paper by Forvi et al. [41] recognises the need for produced MNs to meet both technical and biological standards before being considered safe for human use. The aim of this study was to define an experimental procedure to determine the margin of safety of the $\mathrm{MN}$ array; it is, therefore, an example of a quality control test that could be potentially employed to determine whether produced MNs are mechanically robust enough for application and human use. This paper highlights the importance of testing the MNs and determining if they pass or fail a defined level of quality.

Additionally, many MN arrays are designed for manual insertion by the patient so the range of forces that patients can apply should be taken into account to define the margin of safety. Recently Larrañeta et al. [49] concluded that the range of forces manually applied by 20 volunteers, following instructions for MN insertion, were between 10 and $50 \mathrm{~N}$; the obtained average value was $20 \mathrm{~N}$.

$\mathrm{MN}$ mechanical characterisation tests are commonly employed by $\mathrm{MN}$ groups to demonstrate the mechanical robustness of arrays, therefore allowing judgement of their safety for human use. However, the main issue with $\mathrm{MN}$ mechanical characterisation studies is that a direct comparison cannot be made due to the range of $\mathrm{MN}$ geometrical dimensions, the variety of test protocols employed and the mechanical equipment utilised. In this instance, the $\mathrm{MN}$ area would benefit from consolidation of tests and adoption of a universal, standardised mechanical QCT. 


\section{Techniques Used to Analyse Microneedle Insertion}

There are a number of methods MN groups have employed to demonstrate successful MN insertion with effective reduction in skin barrier function. $\mathrm{MN}$ penetration assessed by staining of $\mathrm{MN}$-created pores, as well as electrical impedance and TEWL monitoring of MN-treated skin, are frequently used to confirm MN-induced skin disruption. Nevertheless, they cannot provide quantitative information on $\mathrm{MN}$ insertion depth, which is considered the most significant determinant for effective transdermal drug permeability $[5,50]$. To overcome the lack of depth information provided by these methods, histological cryosectioning with adjunct staining, confocal microscopy and the novel Optical Coherence Tomography (OCT) have been exploited [5].

\subsection{Staining of Microneedle-Treated Skin}

The use of a coloured dye, such as methylene blue [51,52] or trypan blue $[53,54,55]$, has been widely used by many $\mathrm{MN}$ groups to act as en face visual confirmation of $\mathrm{MN}$ penetration. These dyes selectively stain cells of the viable epidermis and not the SC, therefore successfully identifying MNcreated microchannels [45]. It has been used solely to demonstrate $\mathrm{MN}$ piercing or more commonly used as a quick qualitative test before analysing the skin via a different method to provide more information [5]. Gomaa et al. [53] visualised MN pre-treated skin stained with trypan blue and monitored pre-treated skin via TEWL technology, as illustrated in Figure 6 a. Kochhar et al. [54] used the same dye to stain the perforations and also confirmed pore formation using histological cryosectioning. This post-penetration staining can be used to provide some estimates of the pore diameter by measuring the diameter of the dye spot. It can also be used, as in the Pearton et al. study [52], to visualise the effect of $M N$ insertion force on the microchannels produced; an increase in insertion force causes an increase in skin disruption, resulting in greater permeation of methylene blue [52].

A notable limitation to this method is that lateral diffusion of dyes can lead to overestimation of micropore diameter [56]. Another problem with the method is that sometimes the SC is not truly pierced and methylene blue pools in the indentions creating false positives. An alternative to this method is the injection of the dye into the skin via hollow MNs. Roxhed et al. [48] proposed the injection of a green dye through hollow MN into human volunteers. Similarly, Lee et al. [57] imaged bolus release from dissolving microneedles $(600 \mu \mathrm{m}$ height, $300 \mu \mathrm{m}$ base width, and $600 \mu \mathrm{m}$ centerto-center spacing) in a $6 \times 6$ array. The MNs were inserted by hand into pig cadaver skin. The MNs contained sulforhodamine $B$ at $0.15 \mathrm{wt} \%$ on a dry basis, such that each microneedle contained 0.04 $\mu \mathrm{g}$ of sulforhodamine and the 36-needle array contained $1.44 \mu \mathrm{g}$ of sulforhodamine; the result is illustrated in Figure 6 (b). Following removal, the MN-treated skin was rinsed and thoroughly scrubbed 
with soap solution, thus ensuring the MNs successfully pierced the skin with the dye being injected into the dermis.

\subsection{Electrical Impedance Methods}

Assessing MN piercing of the skin, and the corresponding insertion force required to cause successful penetration, has been investigated by a number of groups through the use of electrical impedance methods $[41,44,48,58,59]$. In this context, impedance is the phase dependant resistance of the skin to the flow of alternating current. The SC represents the main barrier for transdermal delivery of drugs [6]. The electrical impedance technique, Figure 7, exploits the SC's electrical insulating properties to provide information on skin barrier function and confirm if MNs have successfully compromised the SC [48]. A strong inverse correlation between skin permeability and skin electrical impedance has been demonstrated $[58,60]$. Yan et al. [6] recognised a linear relationship between acyclovir flux and the inverse of skin electrical resistance. Typically, the method involves a test/measurement electrode measuring electrical resistance at the site of $\mathrm{MN}$ penetration, while a counter reference electrode is placed nearby to complete the electrical circuit $[58,59]$.

Gupta et al. [58] used this non-invasive technique to investigate both the effect of MN geometry (number, length and cross-sectional area) and occlusion on the resealing properties of the skin in human volunteers. Unlike a number of other papers employing this technique, this study compensates for inter-subject variability and normalises electrical impedance values with the subject's corresponding positive control, hypodermic needle impedance values. The hypodermic needle was a 26 gauge needle inserted $5 \mathrm{~mm}$ into the skin.

The authors commented that a previous insertion study completed by the group involving $350 \mu \mathrm{m}$ MNs failed to produce significant changes in skin impedance, suggesting this method may not have the required sensitivity to detect small electrical changes. In contrast, the sensitivity of the technique was further alluded to by Brogden et al. [59], who commented that this method can detect small changes of electrical impedance in hydrated skin thus indicating that the hydration status of the skin can influence the sensitivity of this technique. They then used this method to demonstrate that micropore lifetime can be extended following $M N$ treatment. Subjects received one-time $M N$ treatment and daily topical application of diclofenac sodium. Micropore closure was measured with using the impedance spectroscopy, and area under the admittance-time curve (AUC) was calculated. AUC was significantly higher at MN + diclofenac sodium sites vs. placebo, suggesting slower rates of micropore healing. This technique and corresponding impedance profiles are illustrated in Figure 7. 


\subsection{Transepidermal Water Loss (TEWL) Monitoring}

Non-invasive TEWL monitoring provides sensitive, instantaneous information on the effects of MN insertion on the skin. Disruption of the skin barrier causes an increase in water loss from the skin's surface (TEWL), which can be utilised in both in vitro and in vivo MN studies [53,61]. TEWL monitoring can determine whether the $\mathrm{SC}$ has been compromised and provides an indirect measure of enhanced skin permeability [51]. The majority of studies document a rapid rise in TEWL values from baseline following MN penetration. Yan et al. [6] recorded an immediate 10-25 fold increase with values returning to baseline over time, indicating skin barrier recovery. This method has been applied to MN experiments investigating the effect of different MN parameters on skin barrier function and also aids in observing the resealing kinetics of the skin's integrity [6,51,53]. Gomaa et al. [53] observed an approximate correlation between the number of trypan-blue stained pores visualised on the MN pretreated skin and TEWL values measured at $1 \mathrm{~h}$ post MN application. They concluded that TEWL values are loosely correlated to the number of $\mathrm{MN}$-induced microchannels produced in the skin.

As with all techniques, there are a number of concerns over the accuracy of TEWL monitoring. Gomaa et al. [53] highlighted an earlier study by Chilcott's group [62] which suggested that TEWL cannot be used to predict transdermal delivery of drugs [63]. No correlation between TEWL and skin integrity was found, however this may be due to the use of different experimental protocols. Variations in TEWL values recorded by different $\mathrm{MN}$ groups are well recognised, which may be due to variation between the skin samples/models used, the lack of universal calibration between equipment, variability between experimental protocols, differences in atmospheric temperatures and the susceptibility for inaccuracies at high vapour flux rates $[53,64]$. TEWL studies also provide no information on the depth of $\mathrm{MN}$ insertion and are highly sensitive to the hydration status of the skin. However, this technique may not be able to detect small changes in water loss in experiments where the skin has been occluded for a number of days [59]. It was also recognised by Yan et al. [6] that there was a difference in recovery between in vitro tests involving human cadaver skin and in vivo rat skin, with the resistance of human cadaver skin remaining constant after $\mathrm{MN}$ pre-treatment representing the lack of skin recovery in this material. This indicates the significance the type of skin sample/model has on the accuracy of studies. Kalluri et al. [51] commented that transdermal drug delivery is enhanced, even following the re-establishment of the skin's water gradient, up until complete restoration of the SC is complete. This raises doubts on the suitability of TEWL methods for measuring MN pore closure, with MN-enhanced skin permeability potentially continuing even after return of the skin's TEWL values to baseline. 


\subsection{Histological Tissue Staining and Sectioning}

Histological cryosectioning is a laborious method which can be used to confirm MN penetration of the skin. Typically the MN-treated skin is excised from the bulk skin sample, fixed in a suitable media, then instantly frozen using liquid nitrogen and stored at $-80^{\circ} \mathrm{C}$ until the time of analysis. A cryostat is used to slice the sample into cryosections of approximately 6-12 $\mu \mathrm{m}$ thick, which are then typically stained with hematoxylin and eosin stains to observe the $\mathrm{MN}$-created microchannels $[47,48,49,65]$. An example of results obtained with this procedure is presented in Figure 8.

There are a number of limitations to this destructive technique. Thus, excising the skin during biopsy and mechanical insults exerted during slicing can potentially affect the hydration status of the skin, as well as causing tension changes within this elastic tissue [66], while removal of the $\mathrm{MN}$ array before addition of the dye can also cause skin retraction and, therefore, pore deformation, which will ultimately affect the measured dimensions of the microconduits [5]. Furthermore, it is often difficult to locate the MN perforation in the skin sample due to the irregular nature of this tissue, as illustrated in the figure. Coulman et al. [56] recognised analysing MN-mediated pore dimensions via histological methods leads to overestimation of measurements. This was highlighted through comparison of histological microchannel images taken in a previous study [67] with OCT images produced by the same type of silicon MNs.

\subsection{Confocal Microscopy}

Confocal laser scanning microscopy has been used to gain valuable knowledge on the dimensions of the $\mathrm{MN}$-created pores $[45,51,68,69]$. Following $M N$ insertion and removal from skin samples, the area is treated with a solution containing specific fluorescent microparticles that migrate down the channels created in the MN breached skin, as in Figure 9. Confocal microscopy can then detect these fluorescence probes, which indicate the depth of the pores [51]. It is a non-invasive technique that provides ex vivo and in vivo images while avoiding the lengthy fixing, sectioning and staining steps required in histology procedures [68]. The major drawback to this method is the fact that confocal microscopy can only penetrate to depths of around 200-250 $\mu \mathrm{m}$ from the skin surface, which limits its potential as a viable technique for measuring the pore dimensions of longer MNs that produce deeper microchannels $[5,68,70]$. Kalluri et al. [51] used this imaging process to determine the depth of the pores produced by the $720 \mu \mathrm{m}$ metal MNs of the commercially available DermaRoller ${ }^{\circledR}$. The results show permeation of the fluorescent FluoSpheres ${ }^{\circledR}$ down the pores to a depth of $152.6 \mu \mathrm{m}$; however this may not actually be the depth of the microchannel but simply the limit of detection for the confocal laser scanning microscope. 
An additional limitation of this method is the required degree of transparency of the MNs. If the MNs are opaque, like silicon, metal or even some coloured polymer MNs, then they have to be removed before imaging. This leads to pore shrinkage and, therefore, underestimation of MN pore dimensions.

\subsection{Optical Coherence Tomography}

Optical Coherence Tomography (OCT) can be described as the optical equivalent to ultrasound, which maps changes of reflected light from a biological tissue as a function of depth $[5,71]$. The ability of OCT to visualise depths of $2000 \mu \mathrm{m}$ demonstrates this technique's superiority over confocal microscopy, which is only capable of penetrating to $200-250 \mu \mathrm{m}$ depths. OCT can therefore be considered as the only technique capable of providing transverse imaging of the SC, epidermis and upper dermis $[5,43,56,68,70]$. OCT is a valuable, non-invasive technique that can be used to observe (Figure 10), in real time, MNs inserted into human subjects' skin to determine the MNs' ability to penetrate the skin [5].

Handheld OCT probes can be particularly useful in vivo for scanning the skin surface. This technique can provide useful information on skin resealing kinetics following $\mathrm{MN}$ removal, in situ dissolution of soluble polymeric MNs $[5,72]$ and has also been utilised for visualising $M N$ penetration into other tissues such as the sclera [73]. Nevertheless, the MNs must be transparent for in situ imaging. In addition, this technique was used by Larrañeta et al. [49] to compare the insertion of polymeric MN arrays in excised neonatal pig skin and an artificial membrane. The superior abilities of this technique are, however, offset to some extent by the expense of the specialist equipment required.

\subsection{Significance of Microneedle Insertion Test Results}

The relevance of $\mathrm{MN}$ studies, and their interpretation, is hindered by a number of factors MNs fabricated from different materials; varying geometries; use of different drugs to investigate permeation; use of varying experimental protocols and different skin models. Such differences presently make it inherently difficult to develop correlation rules [53]. Apart from inter-group variation between the results obtained using similar insertion analysis techniques, variation between results from different tests can exist. This is highlighted by Kalluri et al. [51]. TEWL values suggested MN height has no effect on the rate of micropore closure, with restoration of the skin's barrier function occurring within 4-5 hr following MN treatment. Conversely, parallel pore resealing kinetic studies performed by this group, with the same type of metal MNs but using calcein imaging, found MN height did influence the rate of micropore closure; $770 \mu \mathrm{m} \mathrm{MN}$ pores closed at $18 \mathrm{hr}$ following MN removal in contrast to $12 \mathrm{hr}$ for the $320 \mu \mathrm{m}$ MNs microchannels. This example reinforces the fact that two different methods of analysing MN penetration can have very different results even though the MNs and skin samples used remain the same. 
Conversely, another key aspect that remains largely unexplored in the literature is the design of a standardized release test from MN arrays such as those readily available for traditional dosage forms [74]. Published studies typically use biological tissue for release experiments [75]. However, biological tissue is not suitable for a quality control test as it cannot be standardised. Therefore, an artificial membrane is required that mimics the skin in terms of drug permeation after the insertion of microneedle arrays. Larrañeta et al. [49] recently identified Parafilm $\mathrm{M}^{\circledR}$ as a suitable skin simulant for MN insertion, along with the development of a facile, rapid and reliable insertion test with the potential for use as a QC test method, or for comparative formulation studies, using this material.

\subsection{Computational Modelling}

One method of avoiding these experimental issues is the use of computer modelling whereby various conditions can be simulated potentially reducing the need for experimental work. Software simulation provides the flexibility of altering parameters to investigate a wide range of variables and influencing factors without the need for costly fabrication and experimental processes.

Chiu et al. [76] used dynamic finite element software ANSYS/LS-DYNA to simulate the insertion of PLA MNs into skin to find the optimal design for biodegradable polymer MN patches. The results indicated that the stress distribution of the microneedle becomes larger, by a factor of three, as its base diameter becomes smaller. They caution that a PLA microneedle, if designed with a base diameter lower than $150 \mu \mathrm{m}$, is at risk of fracture. Similarly, Kong et al. [77] investigated the complete insertion process of a $\mathrm{MN}$ into skin to explore the effect of changing $\mathrm{MN}$ geometry on insertion. In contrast though, they focussed on the effect this has on the skin as opposed to the needle itself. In addition, they explored the influence of skin layer thickness, simulating varying anatomical regions, race and age. They concluded that the stratum corneum, needle tip area and wall angle are the primary control parameters for tapered MNs. For hollow microneedles with a large tip diameter, the larger the wall thickness, the larger the insertion force became. For a small tip diameter, the wall thickness had almost no effect on the insertion force. Most importantly, however, the predictions are in agreement with previous experimental work.

Chen et al. [78] also used a nonlinear finite element model based on the microbiomechanical properties of the skin to simulate a microneedle inserting into skin. In this study, the influences of different geometries on $\mathrm{MN}$ fracture were simulated with the intention of optimising $\mathrm{MN}$ design. Experimental validation was completed on mouse skin. For a MN $1000 \mu \mathrm{m}$ long and $100 \mu \mathrm{m}$ wide the simulation predicted that the buckling force would increase with needle angle but be unaffected by tip width. On the other hand, the insertion force would decrease greatly with a decrease in tip width. In particular they concluded that for a MN $1000 \mu \mathrm{m}$ long and $100 \mu \mathrm{m}$ wide, the width of the tip should 
be less than $20 \mu \mathrm{m}$. The results are comparable with their experimental but the simulation over predicted the $\mathrm{MN}$ insertion force. Over predicting insertion force is not disadvantageous as it provides an inherent safety factor but it is not ideal when striving for optimisation of needle geometry; this is particularly the case when over predicted by $40 \%$ as was the case here.

An issue with numerical simulations are the inherent assumptions and difficulties: the skin is a nonlinear material and so does not exhibit ideally elastic behaviour; some models ignore the skin deformation before piercing; changing boundary conditions as contact between microneedle and skin change over time; neglect of the effect of underlying tissues such as muscle and bone and skin layer thicknesses assumed to be identical for all subjects $[78,79]$. These assumptions and difficulties can and do lead to under or overpredictions and as a result can be unreliable.

Groves et al. [79] undertook computer simulation analysis and then used experiemtnal data to refine the computer algorithms in order to eliminate as much error as possible. They completed a similar study to Chen et al. [78] using the same model for simulation in order to predict skin deformation and enable the rational design of optimised MN devices. The difference is that Groves et al. [79] used a material parameter extraction algorithm based on in-vivo indentation experiments to optimise the material coefficients. The Ogden material parameters from the indentation tests were then compared with experimental measurements. Despite the acknowledged limitations, the degree of correspondence between simulation curves and in-vivo data proved to have an accuracy of between 95.1 and $99 \%$. This study highlights the position of computer simulations in practical research as it proves the usefulness of modelling predictions but still the need for experimental data.

\section{Conclusion}

Mechanical characterisation tests act to simulate the types of forces MNs will experience in vivo. They help determine the $\mathrm{MN}$ fracture force and, when considered in combination with the insertion force, can be used to determine a candidate MN system's suitability for human application. The range of MN mechanical characterisation tests, and the experimental equipment used, makes it difficult to directly compare MN's and determine the most suitable array for a given application.

A diverse range of techniques have been utilised in $\mathrm{MN}$ insertion studies. Staining following $\mathrm{MN}$ treatment, electrical impedance and TEWL studies all confirm that the SC has been successfully compromised by the array. Histological sectioning, confocal microscopy and OCT allow depth of MNcreated microchannels to be analysed. Variation in techniques and equipment used, the different procedures followed and the use of varying skin samples/models all make it difficult to compare MN performance. 
MN research would benefit from a select number of universal, standardised QCTs that would allow characterisation and comparison of MN strength and insertion ability. The large-scale manufacturing of MNs will require automated quality QCT in place at each stage of the production line. There are strict regulations and pharmacopoeial standards that a drug product would need to meet in order to be deemed appropriate for release for human use. However, no pharmacopoeial standards will be elaborated for $\mathrm{MN}$-based products until a range of $\mathrm{MN}$ products are marketed, since such tests are derived from those approved by the regulatory authorities as part of a manufacturer's submitted dossier. Thus, the regulatory specifications will ultimately be significantly influenced by the first MN drug delivery products to be marketed, early industry adopters of the technology thus having the advantage of determining the quality standards that later $M N$ products will need to meet $[80,81]$.

The basic requirement for $Q C$ test are to establish the safety MNs as part of a QA system operating within a GMP environment. As a result, the critical questions needing addressed are: What are and how do we determine MN safety and efficacy?

According to the relevant guidelines [33], safety can be addressed from the following but which are relevant to $\mathrm{MN}$ products?

i. Microbial limits;

ii. Sterility;

iii. Particulate matter;

iv. Antimicrobial preservative content;

v. Extractables;

vi. Osmolarity;

Equally how do we define efficacy? Is the following the complete, basic, desirable criteria for MNs? If so, how do we determine these values?

i. Penetrate the skin - Should skin models be used in conjunction with a pre-determined force and rate or is a solid block of a particular material sufficient? What is the pre-determined force and rate? What are the tolerances? What machine and probe should be used; is standardisation of methodology necessary?

ii. Insert to the required depth - What is the required depth? It is known that not the entire length of MN completely inserts into the skin [49] so what is the appropriate depth for a particular array or needle? Is there a required surface area of $\mathrm{MN}$ required to be inserted to achieve maximum delivery of drug cargo? Presumably if there is then as long as this is met there is no need for an upper tolerance. Perhaps, as reported [49], OCT with Parafilm $M^{\circledR}$ may be sufficient for this test. 
iii. MNs do not break whilst in the skin - this will be less important for dissolving microneedles but highly important for solid MNs where there is high fragility and less biocompatibility, such as silicon. Is there a need for torsional tests alongside transverse failure tests or are the penetration test procedures enough to gather relevant data? If these additional tests are required then the same constraints apply as to the penetration tests. What parameters and tolerances, equipment and probes will be suitable? What are the risks and remedies if $\mathrm{MN}$ do break within the skin?

iv. MNs deliver their cargo - arguably the most important requirement alongside skin penetration. How do we ensure the $\mathrm{MN}$ will deliver the cargo in the timeframe required at sufficient therapeutic levels? For hydrogel MNs, swelling may be deemed sufficient; perhaps also in combination with crosslinking degree, i.e., the use of functionality-related characteristics. For dissolving MNs, dissolution/ disintegration tests should be sufficient. For other MN types, presumably it would depend on the method of drug delivery. 'Poke and patch' [18] systems will require analysis of the resulting holes as well as the patch itself. Traditional QC tests for patches will suffice for the latter, while OCT and/or TEWL could be sufficient for the former. For coated needles, or 'coat and poke' [82], some form of release study from the needles will be needed. Finally, for hollow MNs where there will need to be a separate delivery system or injector [83] there will need to be tests to inspect the $\mathrm{MN}$ channels for potential blockage as well as the separate injector system. A further potential test, certainly for transparent or translucent MNs could be a refractive index test. The refractive index is a basic property of polymers which relates to their ability to bend light, it also correlates with other properties of the polymer such as electromagnetic and chemical properties [84] Understanding and developing the criteria to ensure the needles do not get plugged with human tissue will be paramount. The effective delivery of cargo is one of the tests that will more than likely be included in the standards tailored for specific MN types; however, perhaps simple geometry characterisation and OCT + TEWL would be a basic start. Whatever the tests required, there is a need for basic criteria which embraces all MN types at the first step. This first battery of tests can be used universally for research groups and manufacturers alike. It will permit direct comparisons and quick diagnostics of successfully designed and manufactured MNs. Further tests can then be tailored to suit the specificity of the MN category to establish the degree of efficacy and whether this particular design/batch is acceptable. Tolerances must be developed and agreed, with any deviations and out of specification/trend results recorded and investigated.

Above all, all research groups must implement these tests if $\mathrm{MN}$ researchers wish to seriously progress successful, and actually useful, MN research. Hopefully, with a set of universal acceptance criteria and 
development of MN targeted GMP specifications, we can overcome the barriers to manufacturing and thus expand the applicability of TDD far beyond its present limited range of actives, while also achieving a potential shift in areas such as vaccination and diagnostics.

\section{Acknowledgments}

This study was supported by BBSRC grant number BB/K020234/1.

\section{Conflict of Interest}

Ryan Donnelly and David Woolfson are named inventors on a number of patent applications related to microneedle arrays. They are working with a number of companies with a view to commercialisation of technologies in this area. They provide advice, through consultancy, to these companies. This does not alter our adherence to Drug Delivery \& Translational Research policies on sharing data and materials. None of the other authors (Rebecca E.M. Lutton, Eneko Larraneta, Stephen Liggett, Jessica Moore) have any competing interests.

No animal studies were conducted here, since this is a Review Article.

\section{References}

[1] L. Margetts and R. Sawyer. Transdermal drug delivery: principles and opioid therapy. Contin. Educ. Anaesthesia, Crit. Care Pain. 2007; 7(5):171-176.

[2] T. B. Higerd, C. a. Baechler, and R. S. Berk. In Vitro and In Vivo Characterization of Pyocin. J. Bacteriol. 1967; 93:1976-1986.

[3] K. S. Paudel, M. Milewski, C. L. Swadley, N. K. Brogden, P. Ghosh, and A. L. Stinchcomb, Challenges and opportunities in dermal/transdermal delivery. Ther. Deliv. 2010; 1(1):109131.

[4] S. Indermun, R. Luttge, Y. E. Choonara, P. Kumar, L. C. Du Toit, G. Modi, and V. Pillay. Current advances in the fabrication of microneedles for transdermal delivery. J. Control. Release. 2014; 185:130-138

[5] R. F. Donnelly, M. J. Garland, D. I. J. Morrow, K. Migalska, T. R. R. Singh, R. Majithiya, and a D. Woolfson. Optical coherence tomography is a valuable tool in the study of the effects of 
microneedle geometry on skin penetration characteristics and in-skin dissolution. J. Control. Release. 2010; 147(3):333-41.

[6] G. Yan, K. S. Warner, J. Zhang, S. Sharma, and B. K. Gale. Evaluation needle length and density of microneedle arrays in the pretreatment of skin for transdermal drug delivery. Int. J. Pharm. 2010; 391(1-2):7-12.

[7] H. S. Gill and M. R. Prausnitz. Does needle size matter?. J. diabetes Sci. Technol. 2007; $1(5): 725-729$

[8] Y.-C. Kim, J.-H. Park, and M. R. Prausnitz. Microneedles for drug and vaccine delivery. Adv. Drug Deliv. Rev. 2012; 64(14):1547-68.

[9] P. Khanna, J. A. Strom, J. I. Malone, and S. Bhansali. Microneedle-Based Automated Therapy for Diabetes Mellitus. J. Diabetes Sci. Technol. 2008; 2(6):1122-1129.

[10] S.-J. Paik, S. Byun, J.-M. Lim, Y. Park, A. Lee, S. Chung, J. Chang, K. Chun, and D. "Dan” Cho. Inplane single-crystal-silicon microneedles for minimally invasive microfluid systems. Sensors Actuators A Phys. 2004; 114( 2-3):276-284.

[11] R. F. Donnelly, T. Raghu, R. Singh, and A. D. Woolfson, "Microneedle-based drug delivery systems : Microfabrication , drug delivery , and safety. Drug Deliv. 2010; 17(4):187-207.

[12] H. J. G. E. Gardeniers, R. Luttge, E. J. W. Berenschot, M. J. De Boer, S. Y. Yeshurun, M. Hefetz, R. Van Oever, and A. Van Den Berg. Silicon Micromachined Hollow Microneedles for Transdermal Liquid Transport. 2003; 12(6):855-862.

[13] M. A. Ling Teo, C. Shearwood, K. C. Ng, J. Lu, and S. Moochhala. In vitro and in vivo characterization of MEMS microneedles. Biomed. Microdevices. 2005; 7:47-52.

[14] J. Ji, F. E. Tay, J. Miao, and C. Iliescu. Microfabricated Silicon Microneedle Array for Transdermal Drug Delivery. J. Phys. Conf. Ser. 2006; 34:1127-1131.

[15] R. F. Donnelly, D. I. J. Morrow, M. T. C. Mccrudden, A. Z. Alkilani, E. M. Vicente-Pérez, C. O'Mahony, P. González-Vázquez, P.A. McCarron and A. D. Woolfson. Hydrogel-Forming and Dissolving Microneedles for Enhanced Delivery of Photosensitizers and Precursors. Photochem. Photobiol., 2014; 90(3):641-7. 
[16] W. Martanto, J. S. Moore, O. Kashlan, R. Kamath, P. M. Wang, J. M. O'Neal, and M. R. Prausnitz. Microinfusion using hollow microneedles. Pharm. Res. 2006; 23(1); 104-13.

[17] C. J. Martin, C. J. Allender, K. R. Brain, A. Morrissey, and J. C. Birchall. Low temperature fabrication of biodegradable sugar glass microneedles for transdermal drug delivery applications. J. Control. Release. 2012; 158(1):93-101.

[18] W. Martanto, S. P. Davis, N. R. Holiday, J. Wang, H. S. Gill, and M. R. Prausnitz.Transdermal delivery of insulin using microneedles in vivo. Pharm. Res. 2004; 21(6):947-52.

[19] J. Zhu, Q. Shen, Y. Cao, H. Wang, X. Chen, and D. Chen. Characterization of out-of-plane cone metal microneedles and the function of transdermal delivery. Microsyst. Technol. 2012; 19(4):617-621.

[20] A. Trautmann, F. Heuck, C. Mueller, P. Ruther, and O. Paul. Replication of microneedle arrays using vacuum casting and hot embossing. Dig. Tech. Pap. - Int. Conf. Solid State Sensors Actuators Microsystems, TRANSDUCERS. 2005; 2:1420-1423.

[21] R. F. Donnelly, M. T. C. McCrudden, A. Zaid Alkilani, E. Larrañeta, E. McAlister, A. J. Courtenay, M.-C. Kearney, T. R. R. Singh, H. O. McCarthy, V. L. Kett, E. Caffarel-Salvador, S. Al-Zahrani, and A. D. Woolfson. Hydrogel-Forming Microneedles Prepared from 'Super Swelling' Polymers Combined with Lyophilised Wafers for Transdermal Drug Delivery. PLoS One. 2014; 9(10):111547.

[22] K. Tsuchiya, S. Jinnin, H. Yamamoto, Y. Uetsuji, and E. Nakamachi. Design and development of a biocompatible painless microneedle by the ion sputtering deposition method. Precis. Eng. 2010; 34(3):461-466.

[23] J. S. Kochhar, W. J. Goh, S. Y. Chan, and L. Kang. A simple method of microneedle array fabrication for transdermal drug delivery. Drug Dev. Ind. Pharm. 2013; 39(2):299-309.

[24] G. M. Cruise, D. S. Scharp, and J. a Hubbell. Characterization of permeability and network structure of interfacially photopolymerized poly(ethylene glycol) diacrylate hydrogels. Biomaterials. 1998; 19(4):1287-94. 
[25] S. Aoyagi, H. Izumi, Y. Isono, M. Fukuda, and H. Ogawa. Laser fabrication of high aspect ratio thin holes on biodegradable polymer and its application to a microneedle. Sensors Actuators, A Phys. 2007; 139:293-302.

[26] R. F. Donnelly, R. Majithiya, T. R. R. Singh, D. I. J. Morrow, M. J. Garland, Y. K. Demir, K. Migalska, E. Ryan, D. Gillen, C. J. Scott, and a D. Woolfson. Design, optimization and characterisation of polymeric microneedle arrays prepared by a novel laser-based micromoulding technique. Pharm. Res. 2011; 28(1): 41-57.

[27] P. C. DeMuth, Y. Min, B. Huang, J. A. Kramer, A. D. Miller, D. H. Barouch, P. T. Hammond, and D. J. Irvine. Polymer multilayer tattooing for enhanced DNA vaccination. Nat. Mater. 2013; 12(4):367-76.

[28] J. D. Kim, M. Kim, H. Yang, K. Lee, and H. Jung. Droplet-born air blowing: Novel dissolving microneedle fabrication. J. Control. Release. 2013; 170(3); 430-436.

[29] K. L. Yung, Y. Xu, C. Kang, H. Liu, K. F. Tam, S. M. Ko, F. Y. Kwan, and T. M. H. Lee. Sharp tipped plastic hollow microneedle array by microinjection moulding. J. Micromechanics Microengineering, 2012; 22(1):015016.

[30] F. and D. Administration. Guidance for industry: CGMP for phase 1 investigational drugs. MD. 2008. http//www. fda. gov/downloads/Drugs. Accessed 11 Feb 2015.

[31] M. T. C. Mccrudden, A. Z. Alkilani, A. J. Courtenay, C. M. Mccrudden, B. Mccloskey, C. Walker, N. Alshraiedeh, R. E. M. Lutton, B. F. Gilmore, A. D. Woolfson, and R. F. Donnelly. Considerations in the sterile manufacture of polymeric microneedle arrays. Drug Deliv. and Transl. Res. 2014; 5(1):3-14.

[32] European Commission. Chapter 1: Pharmaceutical Quality System Legal. EudraLex Good Manuf. Pract. Guidel. 2013; 4:1-8.

[33] F. and D. Administration. Specifications : Test Procedures And Acceptance Criteria For New Drug Substances And New Drug Products : Q6A. Federal register. 2000; 65(251).

[34] B. Hunter and European Commission. The Rules Governing Medicinal Products in the European Union Volume 4 EU Guidelines for Good Manufacturing Practice for Medicinal Products for 
Human and Veterinary Use Part 1 Chapter 6: Quality Control. Int. J. Integr. Care. 2001; doi: 10.1016/j.nucengdes.2011.01.052.

[35] A. D. W. Eneko Larrañeta, Rebecca E. M. Lutton, Aaron J. Brady, Eva M. Vicente-Pérez. Microwave-assisted preparation of hydrogel-forming microneedle arrays for transdermal drug delivery applications. Macromol. Mater. Eng. 2015; doi: 10.1002/mame.201500016.

[36] European Commission. Working Party on Control of Medicines and Inspections Final Version of Annex 15 to the EU Guide to Good Manufacturing Practice. Medicinal Products For Human Use. 2015. http://ec.europa.eu/health/files/eudralex/vol-4/2015-10_annex15.pdf. Accessed 11 Feb 2015.

[37] European Commission. EudraLex The Rules Governing Medicinal Products in the European Union Volume 4 EU Guidelines to Good Manufacturing Practice Medicinal Products for Human and Veterinary Use Annex 1 Manufacture of Sterile Medicinal Products (corrected version). News And Updates On Pharmaceuticals. 2008. http://ec.europa.eu/health/files/eudralex/vol4/2015-10_annex15.pdf. Accessed 11 Feb 2015.

[38] P. Khanna, K. Luongo, J. a Strom, and S. Bhansali. Sharpening of hollow silicon microneedles to reduce skin penetration force. J. Micromechanics Microengineering. 2010; 20:045011.

[39] P. Khanna, H. Silva, and S. Bhansali. Variation in microneedle geometry to increase shear strength. Procedia Eng. 2010; 5:977-980.

[40] J. Zahn, N. Talbot, D. Liepmann, and A. Pisano. Microfabricated polysilicon microneedles for minimally invasive biomedical devices. Biomed. Microdevices. 2000; 2(4):295-303.

[41] E. Forvi, M. Soncini, M. Bedoni, F. Rizzo, M. Casella, C. O’Mahony, and F. Gramatica. A method for determine the margin of safety for microneedles arrays. ICME'10 The 2010 International Conference of Mechanical Engineering, London, U.K., 30 June - 2 July 2010.

[42] J.-H. Park, M. G. Allen, and M. R. Prausnitz. Biodegradable polymer microneedles: fabrication, mechanics and transdermal drug delivery. J. Control. Release. 2005; 104(1):51-66.

[43] Y. K. Demir, Z. Akan, and O. Kerimoglu. Characterization of Polymeric Microneedle Arrays for Transdermal Drug Delivery. PLoS One. 2013; 8(10):1-9. 
[44] S. P. Davis, B. J. Landis, Z. H. Adams, M. G. Allen, and M. R. Prausnitz. Insertion of microneedles into skin: measurement and prediction of insertion force and needle fracture force. J. Biomech. 2004; 37(8):1155-63.

[45] S. D. Gittard, B. Chen, H. Xu, A. Ovsianikov, B. N. Chichkov, N. a Monteiro-Riviere, and R. J. Narayan. The Effects of Geometry on Skin Penetration and Failure of Polymer Microneedles. J. Adhes. Sci. Technol. 2013; 27:227-243.

[46] Jeong Woo Lee, Mee-Ree Han, Jung-Hwan Park. Polymeric microneedles for transdermal drug delivery. Journal of Drug Targeting. 2013; 21(3):211-223.

[47] P. Loeters, R. Duwel, F. Verbaan, R. Luttge, D. van den Berg, J. Bouwstra, and A. van den Berg. Measuring the insertion ofmicrofabri- cated microneedles into skin with a penetration sensor. Proc. TTAS 8th Int. Conf.Miniaturized Syst. Chem. Life Sci. 2004; 1:497-499.

[48] N. Roxhed, T. C. Gasser, P. Griss, G. a. Holzapfel, and G. Stemme. Penetration-enhanced ultrasharp microneedles and prediction on skin interaction for efficient transdermal drug delivery. J. Microelectromechanical Syst. 2007; 16(6):1429-1440.

[49] E. Larrañeta, J. Moore, E. M. Vicente-Pérez, P. González-Vázquez, R. Lutton, A. D. Woolfson, and R. F. Donnelly. A proposed model membrane and test method for microneedle insertion studies. Int. J. Pharm. 2014; 472(1-2):65-73.

[50] A. Davidson, B. Al-Qallaf, and D. B. Das. Transdermal drug delivery by coated microneedles: Geometry effects on effective skin thickness and drug permeability. Chem. Eng. Res. Des. 2008; 86:1196-1206.

[51] H. Kalluri, C. S. Kolli, and A. K. Banga. Characterization of microchannels created by metal microneedles: formation and closure. AAPS J. 2011; 13(3):473-481.

[52] M. Pearton, V. Saller, S. a. Coulman, C. Gateley, A. V. Anstey, V. Zarnitsyn, and J. C. Birchall. Microneedle delivery of plasmid DNA to living human skin: Formulation coating, skin insertion and gene expression. J. Control. Release. 2012; 160(3):561-569.

[53] Y. a Gomaa, D. I. J. Morrow, M. J. Garland, R. F. Donnelly, L. K. El-Khordagui, and V. M. Meidan. Effects of microneedle length, density, insertion time and multiple applications on 
human skin barrier function: assessments by transepidermal water loss. Toxicol. In Vitro, 2010; 24(7):1971-8.

[54] J. S. Kochhar, T. C. Quek, W. J. Soon, J. Choi, S. Zou, and L. Kang. Effect of microneedle geometry and supporting substrate on microneedle array penetration into skin. J. Pharm. Sci., 2013; 102(11):4100-8.

[55] D. V McAllister, P. M. Wang, S. P. Davis, J.-H. Park, P. J. Canatella, M. G. Allen, and M. R. Prausnitz. Microfabricated needles for transdermal delivery of macromolecules and nanoparticles: fabrication methods and transport studies. Proc. Natl. Acad. Sci. U. S. A.. 2003; 100(24):13755-60.

[56] P. B. Coulman SA, Birchall JC, Alex A, Pearton M, Hofer B, O'Mahony C, Drexler W. In vivo, in situ imaging of microneedle insertion into the skin of human volunteers using optical coherence tomography. Pharm. Res. 2011; 28:66-81.

[57] J. W. Lee, J.-H. Park, and M. R. Prausnitz. Dissolving microneedles for transdermal drug delivery. Biomaterials, 2008; 29(13):2113-24.

[58] J. Gupta, H. S. Gill, S. N. Andrews, and M. R. Prausnitz. Kinetics of skin resealing after insertion of microneedles in human subjects. J. Control. Release. 2011; 154(2):148-155.

[59] N. K. Brogden, M. Milewski, P. Ghosh, L. Hardi, L. J. Crofford, and A. L. Stinchcomb. Diclofenac delays micropore closure following microneedle treatment in human subjects. J. Control. Release. 2012; 163:220-229.

[60] P. Karande, A. Jain, and S. Mitragotri. Relationships between skin's electrical impedance and permeability in the presence of chemical enhancers. J. Control. Release. 2006; 110:307-313.

[61] S. M. Bal, J. Caussin, S. Pavel, and J. a. Bouwstra. In vivo assessment of safety of microneedle arrays in human skin. Eur. J. Pharm. Sci. 2008; 35:193-202.

[62] R. P. Chilcott, C. H. Dalton, A. J. Emmanuel, C. E. Allen, and S. T. Bradley, "Transepidermal water loss does not correlate with skin barrier function in vitro. J. Invest. Dermatol. 2002; 118: 871-875.

[63] Verbaan FJ, Bal SM, van den Berg DJ, Groenink WH, Verpoorten H, Lüttge R, Bouwstra JA. Assembled microneedle arrays enhance the transport of compounds varying over a large 
range of molecular weight across human dermatomed skin. J. Control. Release. 2007; $117: 238-245$.

[64] E. Elmahjoubi, Y. Frum, G. M. Eccleston, S. C. Wilkinson, and V. M. Meidan. Transepidermal water loss for probing full-thickness skin barrier function: Correlation with tritiated water flux, sensitivity to punctures and diverse surfactant exposures. Toxicol. Vitr. 2009; 23(7):1429-1435.

[65] L. Y. Chu and M. R. Prausnitz. Separable arrowhead microneedles. J. Control. Release. 2001; 149(3):242-249.

[66] C. Pailler-Mattei, S. Bec, and H. Zahouani. In vivo measurements of the elastic mechanical properties of human skin by indentation tests. Med. Eng. Phys. 2008; 30:599-606.

[67] J. Birchall, S. Coulman, M. Pearton, C. Allender, K. Brain, A. Anstey, C. Gateley, N. Wilke, and A. Morrissey. Cutaneous DNA delivery and gene expression in ex vivo human skin explants via wet-etch micro-fabricated micro-needles. J. Drug Target. 2005; 13:415-421.

[68] Fábia Cristina Rossetti, Lívia Vieira Depieri and Maria Vitória Lopes Badra Bentley. Confocal Laser Scanning Microscopy as a Tool for the Investigation of Skin Drug Delivery Systems and Diagnosis of Skin Disorders. In: Confocal Laser Microscopy - Principles and Applications in Medicine, Biology, and the Food Sciences. In: INTECH. 2013. http://www.intechopen.com/books/confocal-laser-microscopy-principles-and-applicationsin-medicine-biology-and-the-food-sciences/confocal-laser-scanning-microscopy-as-a-tool-forthe-investigation-of-skin-drug-delivery-systems-and. Accessed 11 Feb 2015.

[69] S. Bal, a. C. Kruithof, H. Liebl, M. Tomerius, J. Bouwstra, J. Lademann, and M. Meinke, "In vivo visualization of microneedle conduits in human skin using laser scanning microscopy. Laser Phys. Lett. 2010; 7(3):242-246.

[70] A. F. Fercher. Optical coherence tomography - development, principles, applications. Z. Med. Phys. 2010; 20:251-76.

[71] M. Mogensen, L. Thrane, T. M. Joergensen, P. E. Andersen, and G. B. E. Jemec. Optical Coherence Tomography for Imaging of Skin and Skin Diseases. Semin. Cutan. Med. Surg. 2009; 28(3):196-202. 
[72] A. Pattani, P. F. McKay, M. J. Garland, R. M. Curran, K. Migalska, C. M. Cassidy, R. K. Malcolm, R. J. Shattock, H. O. McCarthy, and R. F. Donnelly. Microneedle mediated intradermal delivery of adjuvanted recombinant HIV-1 CN54gp140 effectively primes mucosal boost inoculations. J. Control. Release. 2012; 162(3):529-37.

[73] R. R. S. Thakur, S. J. Fallows, H. L. Mcmillan, R. F. Donnelly, and D. S. Jones. Microneedlemediated intrascleral delivery of in situ forming thermoresponsive implants for sustained ocular drug delivery. J. Pharm. Pharmacol. 2014; 66(4):584-95.

[74] M. Siewert, J. Dressman, C. K. Brown, and V. P. Shah. FIP/AAPS guidelines to dissolution/in vitro release testing of novel/special dosage forms. AAPS PharmSciTech. 2003;4(1):E7.

[75] M. J. Garland, K. Migalska, T.-M. Tuan-Mahmood, T. Raghu Raj Singh, R. Majithija, E. CaffarelSalvador, C. M. McCrudden, H. O. McCarthy, a David Woolfson, and R. F. Donnelly. Influence of skin model on in vitro performance of drug-loaded soluble microneedle arrays. Int. J. Pharm. 2012;434(1-2):80-9.

[76] C. Y. Chiu, H. C. Kuo, Y. Lin, J. L. Lee, Y. K. Shen, and S. J. Kang. Optimal Design of Microneedles Inserts into Skin by Numerical Simulation. Key Eng Mat. 2012;516:624-628.

[77] X. Q. Kong, P. Zhou, and C. W. Wu. Numerical simulation of microneedles' insertion into skin. Comput. Methods Biomech. Biomed. Engin. 2011;14(9):827-35.

[78] J. Chen, N. Li, and S. Chen. Finite element analysis of microneedle insertion into skin. Micro Nano Lett. 2012;7(12):1206-1209.

[79] R. B. Groves, S. a Coulman, J. C. Birchall, and S. L. Evans. Quantifying the mechanical properties of human skin to optimise future microneedle device design. Comput. Methods Biomech. Biomed. Engin.2012;15(1):73-82.

[80] A. D. W. Ryan F. Donnelly, Thakur Raghu Raj Singh, Desmond I. J. Morrow. Microneedlemediated Transdermal and Intradermal Drug Delivery. 1st ed. Wiley-Blackwell. 2012.

[81] R. F. Donnelly and A. D. Woolfson. Patient safety and beyond: what should we expect from microneedle arrays in the transdermal delivery arena?. Ther. Deliv. 2014;5(6):653-662.

[82] M. R. Prausnitz. Microneedles for transdermal drug delivery. Adv. Drug Deliv. Rev. 2004;56(5):581-7. 
[83] F. J. Verbaan, S. M. Bal, D. J. van den Berg, J. a. Dijksman, M. van Hecke, H. Verpoorten, a. van den Berg, R. Luttge, and J. a. Bouwstra. Improved piercing of microneedle arrays in dermatomed human skin by an impact insertion method. J. Control. Release. 2008;128:8088.

[84] Ololade Olatunji, Chima C. Igwe, Aroke S. Ahmed, Dewale O. A. Alhassan, GGodfrey O. Asieba, Bhusan Das Diganta. Microneedles from Fish Scale Biopolymer. J. Appl. Polym. Sci. 2014;131(12):40377. 


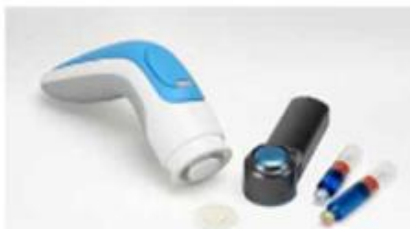

A
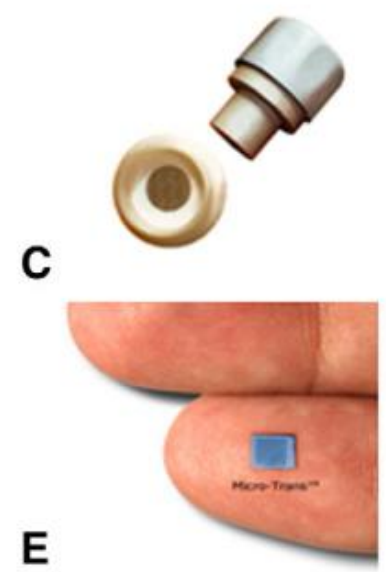

E



G
H
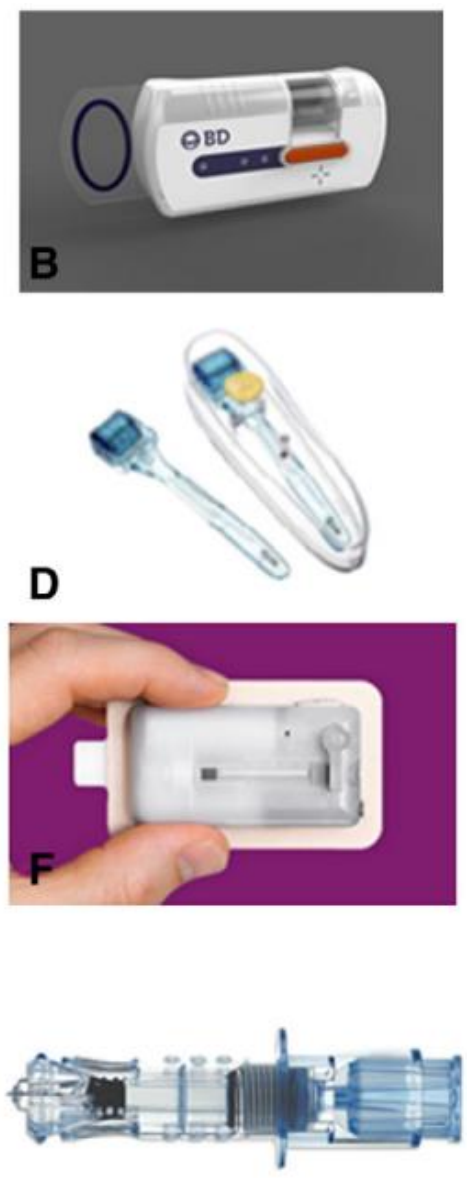

Figure 1. Current microneedle devices. A) Microstructured Transdermal System (MTS). B) Microinfusor. C) Macroflux ${ }^{\circledR}$. D) Microneedle Therapy System (MTS Roller ${ }^{\mathrm{TM}}$ ). E) Microtrans ${ }^{\mathrm{TM}}$. F) hpatch $^{\text {TM }}$. G) MicronJet. H) Intanza ${ }^{\circledR}$. Reproduced with permission from Indermun et al. [4]. 

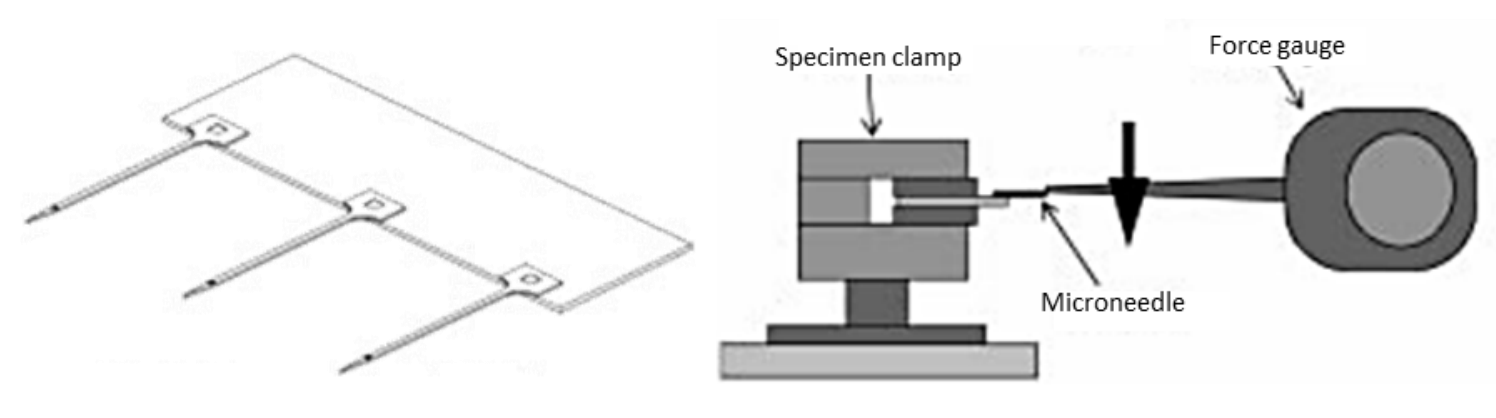

Figure 2. Experimental setup used by Zahn et al. The left image shows the individual polysilicon MNs attached to the glass slide while the right image illustrates the force gauge applying a vertical force to the single MNs. Reproduced with permission from Zahn et al.[40]. 

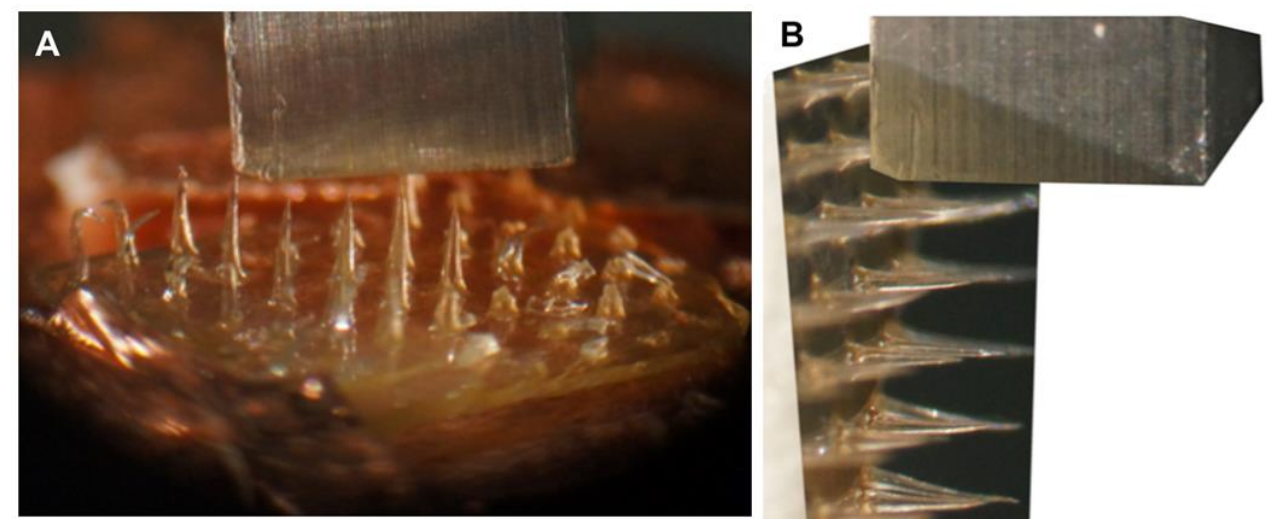

Figure 3. Digital photograph of $M N$ pressed against the metal mill during axial fracture force (A). $M N$ shafts were transversely pressed against the metal mill for measurement of the transverse fracture force (B). Taken from Demir et al. [43]. 

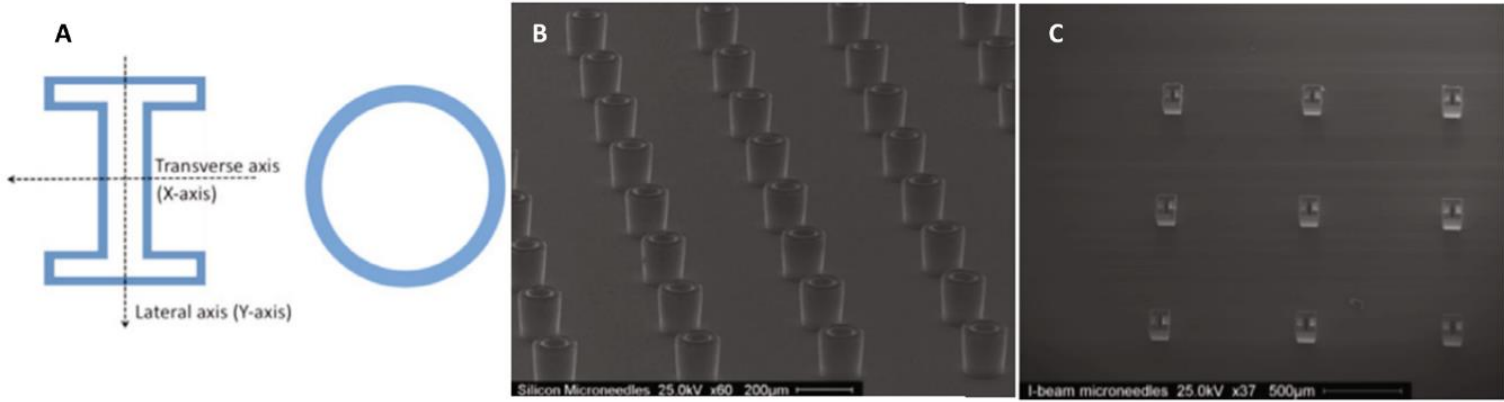

Figure 4. Cross-sectional illustration of the produced MNs (A). Scanning Electron Microscope of circular lumen MNs (B) and 'I-shaped' lumen MNs (C). Reproduced with permission from Khann et al. [39]. 


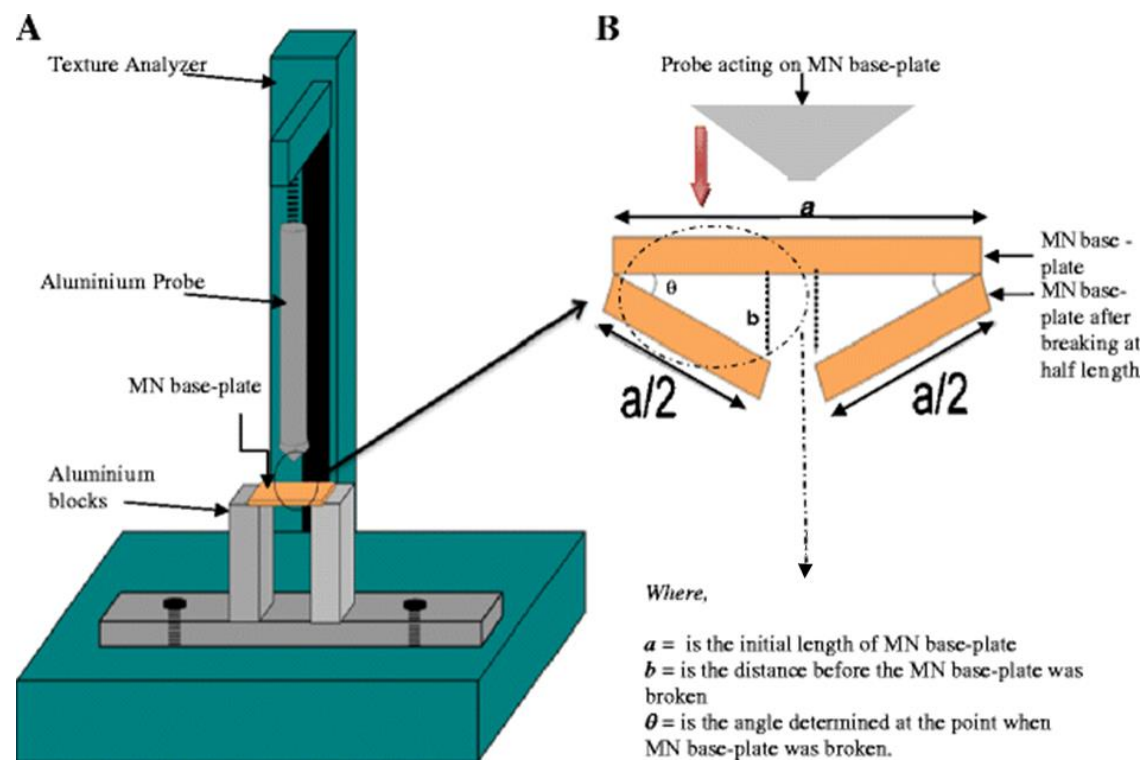

Figure 5. Illustration of the Texture Analyzer set-up (A); the method employed in measuring the degree of flexibility of MN base-plates (B). Reproduced with permission from Donnelly et al. [26]. 


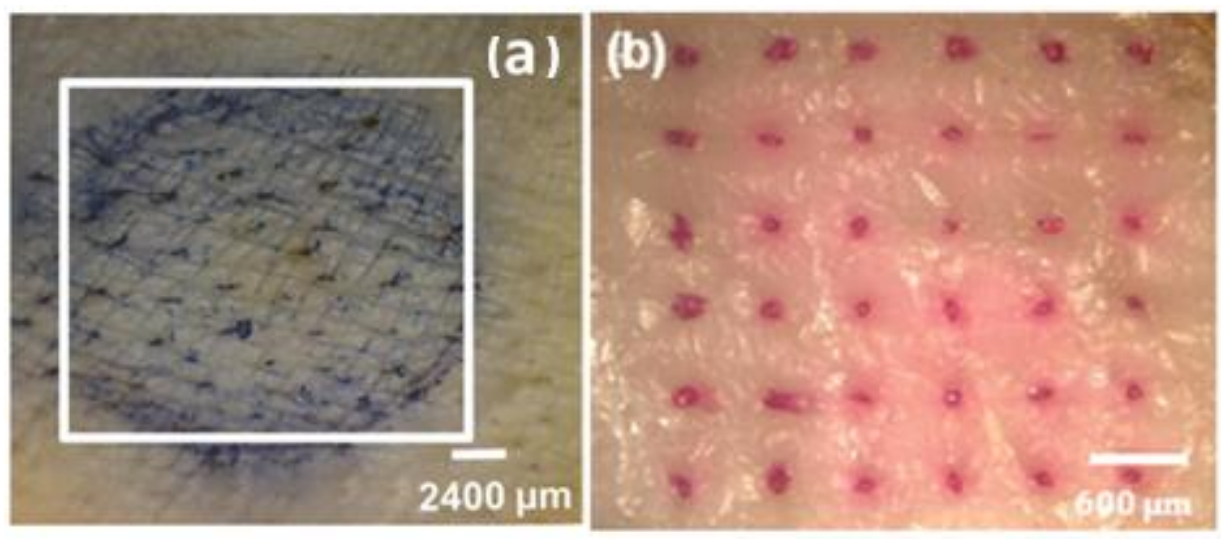

Figure 6. Microneedle penetration performed on pig ear skin for $400 \mu \mathrm{m}$ base diameter, $2400 \mu \mathrm{m}$ center-to-center spacing microneedles (A). Reproduced with permission from Kochhar et al. [54]. Skin surface showing sulforhodamine delivered into the skin by insertion of Dissolving microneedles for bolus delivery into skin (B). Reproduced with permission from Lee et al. [57]. 



Figure 7. Treatment patches and electrodes on a subject's upper arm. The reference electrode was placed in the middle of all the treatment sites; both the reference and measurement electrodes are connected by lead wires to the impedance meter (A). Representative profiles of micropore admittance from two subjects (B) and (C). Values were normalized to the highest post-MN admittance value. Two independent sites were treated with $\mathrm{MN}+$ diclofenac (solid lines with solid shapes), and two additional sites were treated with $\mathrm{MN}+$ placebo (dashed lines with open shapes). Despite differences in the shape of the profiles at diclofenac sites, all placebo treatment sites follow an approximately exponential decay. Reproduced with permission from Brogden et al. [59]. 

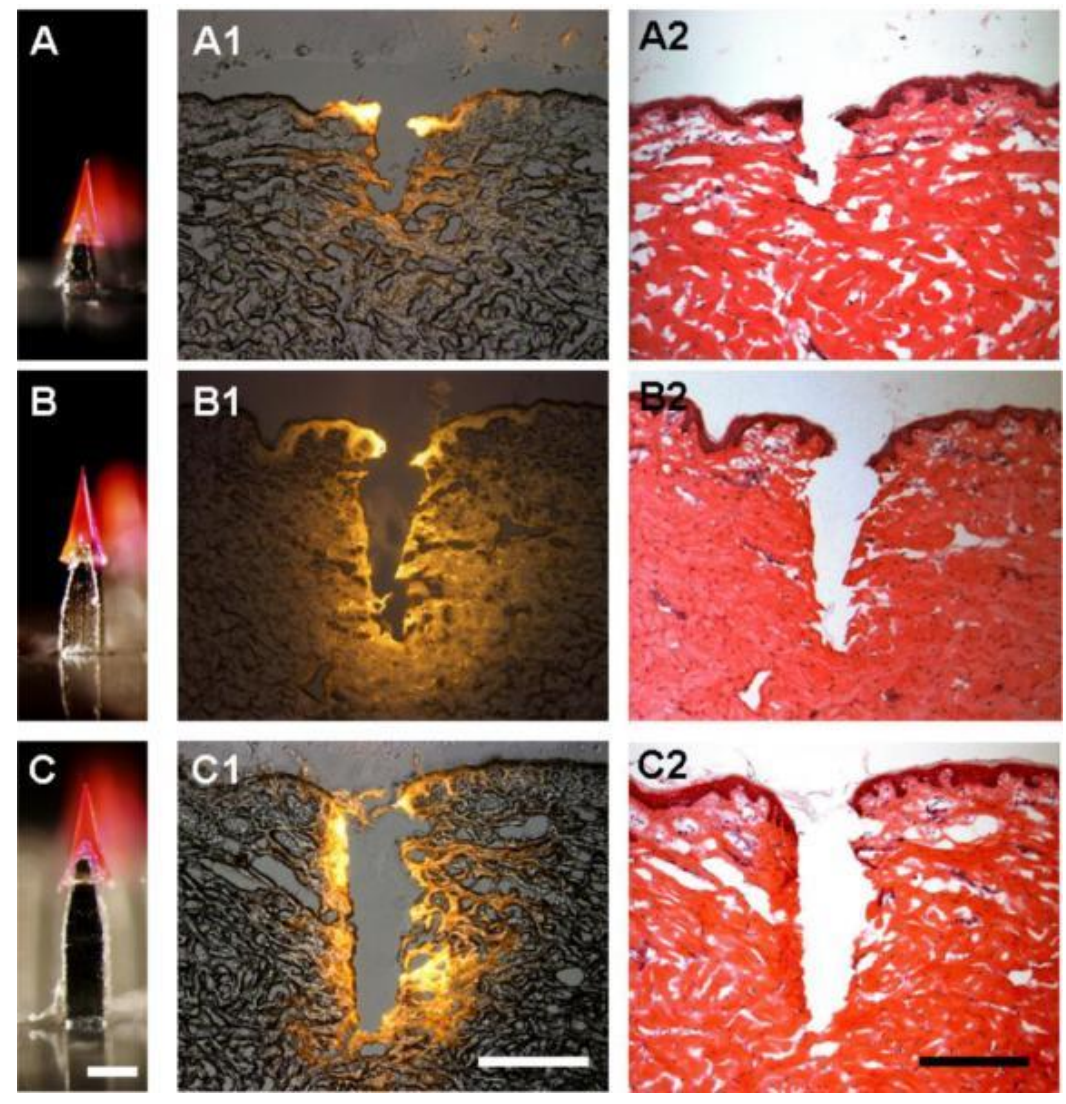

Figure 8. The effect of microneedle shaft length on arrowhead insertion depth into porcine cadaver skin. Arrowheads measuring $600 \mu \mathrm{m}$ tall and encapsulating sulforhodamine mounted on shafts measuring $300 \mu \mathrm{m}(\mathrm{A}), 600 \mu \mathrm{m}$ (B) and $900 \mu \mathrm{m}$ (C) were inserted into skin, which was imaged after histological sectioning by fluorescence microscopy $(A 1, B 1, C 1)$ and $H \& E$ staining $(A 2, B 2, C 2)$. Bar = $300 \mu \mathrm{m}$. Reproduced with permission from L. Y. Chu and M. R. Prausnitz [65]. 


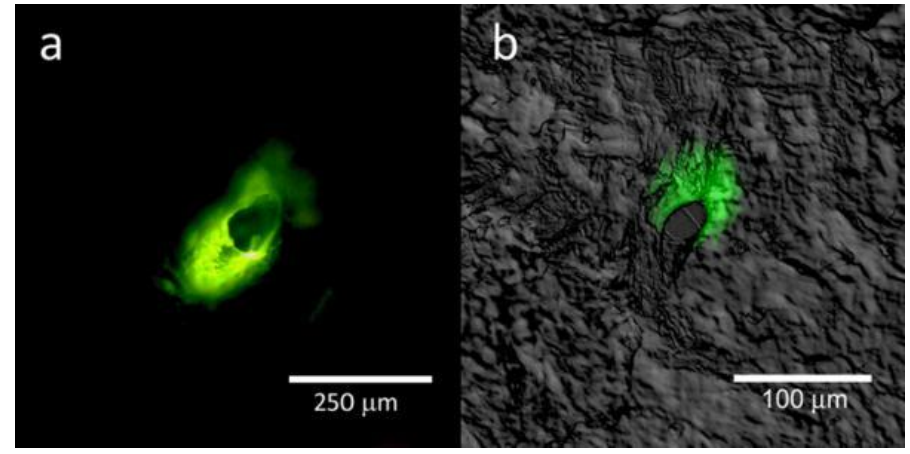

Figure 9. Fluorescence microscopy images of lucifer yellow delivery on the porcine skin surface; confocal and differential interference contrast overlay data of lucifer yellow delivery at a depth of 120 $\mu \mathrm{m}$ are shown. The microneedle-produced pores were noted to possess irregular shapes. In addition, delivery of the lucifer yellow dye into the surrounding region of the skin was noted. Reproduced with permission from Gittard et al. [45]. 


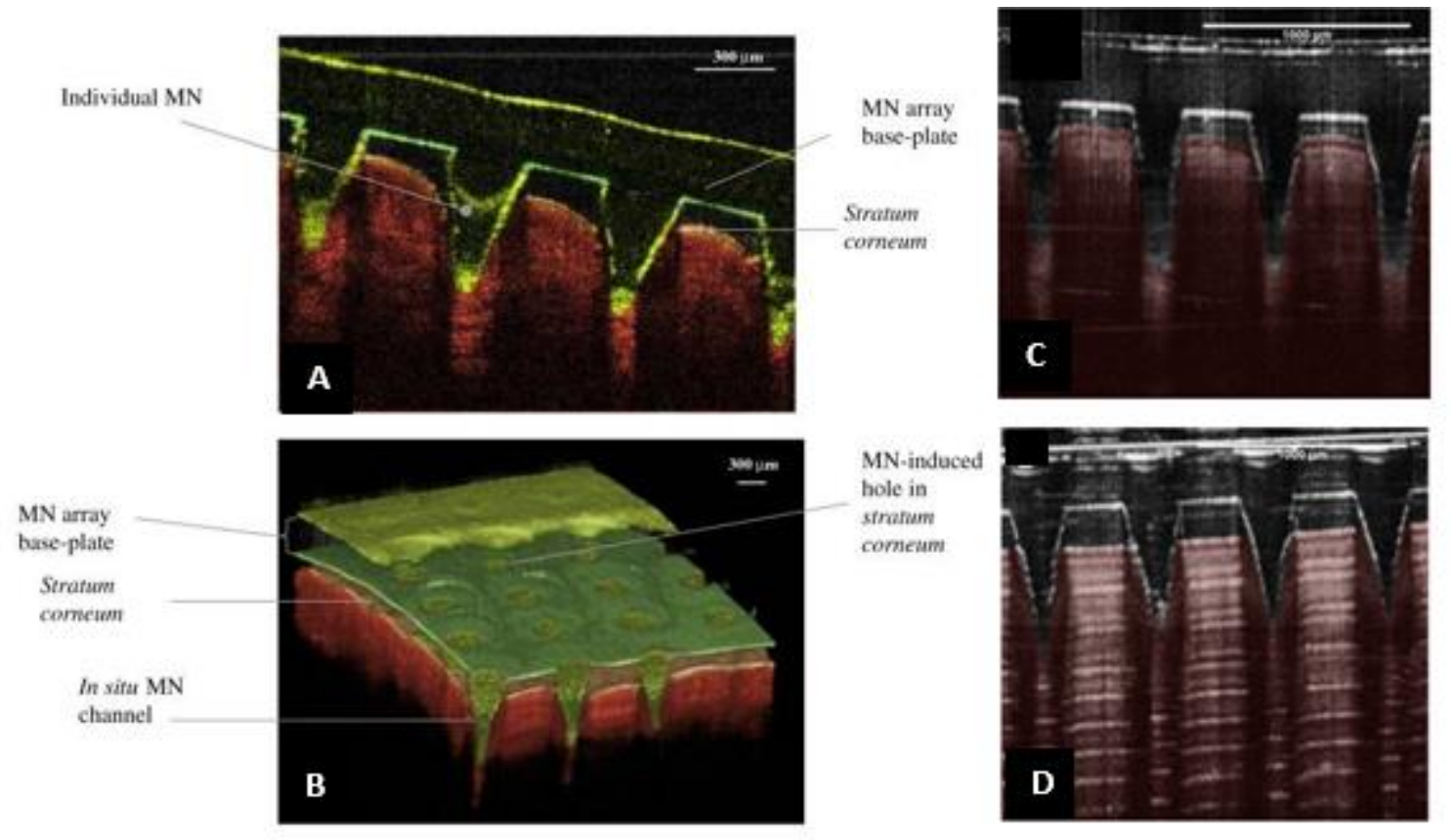

Figure 10. OCT images showing MN (height $600 \mu \mathrm{m}$, width at base $300 \mu \mathrm{m}$, spacing $300 \mu \mathrm{m}$ ) inserted into human skin in vivo in 2D (A); 3D (B). Reproduced with permission from Donnelly et al. [25]. OCT images of a $11 \times 11 \mathrm{MN}$ array inserted manually by the same volunteer in different materials: neonatal pig skin (C); eight layers of PF (D). To allow differentiation between MNs a red false colour were applied in the skin/film layers [49]. 